\title{
MORIHIKO SAITO
}

\section{Induced $\mathcal{D}$-modules and differential complexes}

Bulletin de la S. M. F., tome 117, no 3 (1989), p. 361-387

<http://www.numdam.org/item?id=BSMF_1989_117_3_361_0>

(C) Bulletin de la S. M. F., 1989, tous droits réservés.

L'accès aux archives de la revue «Bulletin de la S. M. F. » (http: //smf.emath.fr/Publications/Bulletin/Presentation.html) implique l'accord avec les conditions générales d'utilisation (http://www.numdam.org/ conditions). Toute utilisation commerciale ou impression systématique est constitutive d'une infraction pénale. Toute copie ou impression de ce fichier doit contenir la présente mention de copyright.

\section{Numdam}




\title{
INDUCED $\mathcal{D}$-MODULES AND DIFFERENTIAL COMPLEXES
}

\author{
BY
}

MoRIHIKo SAITO $(*)$

RÉSuMÉ. - On introduit la notion de complexe différentiel et de $\mathcal{D}$-Module induit, on définit leur dual et image directe et on démontre la dualité pour un morphisme propre, ce qui implique la dualité de $\mathcal{D}$-Modules avec la compatibilité à celle de Verdier. On donne aussi une remarque sur la preuve de la correspondance de Riemann-Hilbert.

Abstract. - We introduce the notion of differential complex and induced $\mathcal{D}$-Module, define their duals and direct images, and prove the duality for proper morphisms, which implies the duality of $\mathcal{D}$-Modules and its compatibility with the Verdier duality. A remark on the proof of the Riemann-Hilbert correspondence is also given.

\section{Introduction}

Let $f: X \rightarrow Y$ be a proper morphism of complex manifolds, or smooth algebraic varieties, and $M^{\bullet} \in D_{\text {coh }}^{b}\left(\mathcal{D}_{X}\right)$ a bounded complex of $\mathcal{D}_{X}$-Modules with coherent cohomologies. Then we have the duality isomorphism ( $c f$. also [B, Be, Sc1-2]) :

$$
f_{*} \mathbb{D} M^{\bullet} \stackrel{\sim}{\longrightarrow} \mathbb{D} f_{*} M^{\bullet} \quad \text { in } D_{\mathrm{coh}}^{b}\left(\mathcal{D}_{Y}\right),
$$

if $\mathcal{H}^{j} M^{\bullet}$ have good filtrations (locally on $Y$ ). Here $\mathbb{D}$ is the dual functor, and $f_{*}$ is the direct image of $\mathcal{D}$-Modules. For simplicity, assume $X=\mathbb{P}^{n}$, $Y=\mathrm{pt}$, and $M^{j}$ are direct sums of $\mathcal{D}_{X} \otimes_{\mathcal{O}_{X}} \mathcal{O}_{X}(p)$. Then we have the isomorphism (0.1) for each $M^{j}$ by the Serre duality, but it is not completely trivial that the differentials on the both sides of $(0.1)$ commute with the isomorphism, because we have to prove some relation between the duality isomorphism and the action of differential operators, $e . g$. the action of the global vector fields on $H^{n}\left(\mathbb{P}^{n}, \omega_{\mathbb{P}^{n}}^{n}\right)$ is zero (this can be easily

(*) Texte reçu le 2 octobre 1988 , révisé le 10 mars 1989

M. SAito, R.I.M.S., Kyoto Univ., Kyoto 606, Japan et I.A.S., Princeton, N.J. 08540, U.S.A. 
checked, if the distributions are used in the Serre duality). In this note we show that the duality isomorphism (0.1) is naturally defined in general by introducing the notion of induced $\mathcal{D}$-Module and differential complexe.

An induced $\mathcal{D}_{X}$-Module $M$ is a right $\mathcal{D}_{X}$-Module isomorphic to $L \otimes_{\mathcal{O}_{X}} \mathcal{D}_{X}$ for an $\mathcal{O}_{X}$-Module $L$. Then we have $f_{*} M \simeq \mathbb{R} f_{\bullet} L \otimes_{\mathcal{O}_{Y}} \mathcal{D}_{Y}$ by definition, where $f_{\text {}}$ denotes the sheaf theoretic direct image, and $f$ is always assumed to be proper. The dual $\mathbb{D} M$ of $M$ is defined by

$$
\mathbb{D} M=\mathbb{R} \mathcal{H o m}_{\mathcal{O}_{X}}\left(L, \omega_{X}\left[d_{X}\right]\right) \otimes_{\mathcal{O}_{X}} \mathcal{D}_{X}
$$

This definition coincides with the usual one,

$$
\mathbb{R} \mathcal{H o m}_{\mathcal{D}_{X}}\left(L \otimes \mathcal{D}_{X}, \omega_{X}\left[d_{X}\right] \otimes D_{X}\right),
$$

if $L$ is coherent. Then the duality isomorphism (0.1) for such $M$ is defined by

$$
\begin{aligned}
f_{*} \mathbb{D} M= & \mathbb{R} f_{\bullet} \mathbb{R} \mathcal{H o m}_{\mathcal{O}_{X}}\left(L, \omega_{X}\left[d_{X}\right]\right) \otimes_{\mathcal{O}_{Y}} \mathcal{D}_{Y} \\
\longrightarrow & \mathbb{R} \mathcal{H o m}_{\mathcal{O}_{Y}}\left(\mathbb{R} f_{\bullet} L, \mathbb{R} f_{\bullet} \omega_{X}\left[d_{X}\right]\right) \otimes_{\mathcal{O}_{Y}} \mathcal{D}_{Y} \\
& \stackrel{\operatorname{Tr}_{f}}{\longrightarrow} \mathbb{R} \mathcal{H o m}_{\mathcal{O}_{Y}}\left(\mathbb{R} f_{\bullet} L, \omega_{Y}\left[d_{Y}\right]\right) \otimes_{\mathcal{O}_{Y}} \mathcal{D}_{Y}=\mathbb{D} f_{*} M
\end{aligned}
$$

using the analytic, or algebraic, trace morphism $\operatorname{Tr}_{f}: \mathbb{R} f_{\bullet} \omega_{X}\left[d_{X}\right] \rightarrow$ $\omega_{Y}\left[d_{Y}\right]$, where $\omega_{X}=\Omega_{X}^{d_{X}}$ and $d_{X}=\operatorname{dim} X$. But we have to still impose some condition on the trace morphism for the compatibility of the morphism (0.3) with the differential of $M$, if $M$ becomes a complex, and this condition is rather difficult to satisfy in the level of complex, $c f .3 .14$. This point can be further simplified by using the associated differential complexes.

For $\mathcal{O}_{X}$-Modules $L, L^{\prime}$ the differential morphisms of $L$ to $L^{\prime}$ are the image of the injective morphism defined by $\otimes_{\mathcal{D}_{X}} \mathcal{O}_{X}$ :

$$
\mathcal{H o m}_{\mathcal{D}_{X}}\left(L \otimes \mathcal{D}_{X}, L^{\prime} \otimes \mathcal{D}_{X}\right) \longrightarrow \mathcal{H o m}_{\mathbb{C}_{X}}\left(L, L^{\prime}\right)
$$

where the image is denoted by $\mathcal{H o m}_{\text {Diff }}\left(L, L^{\prime}\right)$. This injectivity means that the morphisms of the induced Modules are completely recovered by the associated differential morphisms. The direct image of $L$ is defined simply by the sheaf theoretic direct image, i.e. the differential morphisms are stable by the direct image. Then the above duality isomorphism (0.3) corresponds to

$$
\begin{aligned}
f_{\bullet} \mathcal{H o m}_{\text {Diff }}^{f}\left(L, \widetilde{K}_{X}\right) \longrightarrow \mathcal{H o m}_{\text {Diff }}^{f}\left(\mathbb{R} f_{\bullet} L, f_{\bullet} \widetilde{K}_{X}\right) \\
\stackrel{\operatorname{Tr}_{f}}{\longrightarrow} \mathcal{H o m}_{\text {Diff }}^{f}\left(\mathbb{R} f_{\bullet} L, \widetilde{K}_{Y}\right)
\end{aligned}
$$

TOME $117-1989-\mathrm{N}^{\circ} 3$ 
where $\widetilde{K}_{X}=D R\left(K_{X}\right)$ with $\omega_{X}\left[d_{X}\right] \rightarrow K_{X}$ an injective resolution as $\mathcal{D}_{X^{-}}$ Modules, and $\mathcal{H o m}_{\text {Diff }}^{f}$ denotes the subsheaf of $\mathcal{H}_{\text {om }}$ Diff corresponding to

$$
\begin{aligned}
\bigcup_{p} \mathcal{H o m}_{\mathcal{O}_{X}}\left(L, L^{\prime} \otimes F_{p} \mathcal{D}_{X}\right) \hookrightarrow \mathcal{H o m}_{\mathcal{O}_{X}}\left(L, L^{\prime} \otimes \mathcal{D}_{X}\right) \\
\quad=\mathcal{H o m}_{\mathcal{D}_{X}}\left(L \otimes \mathcal{D}_{X}, L^{\prime} \otimes \mathcal{D}_{X}\right) .
\end{aligned}
$$

Here the trace morphism $\operatorname{Tr}_{f}: f_{\bullet} \widetilde{K}_{X} \rightarrow \widetilde{K}_{Y}$ is defined to be compatible with the filtration $F$ associated to the de Rham functor $D R$, and $\operatorname{Gr}_{0}^{F} \operatorname{Tr}_{f}$ coincides with the above analytic, or algebraic, trace morphism $\operatorname{Tr}_{f}$ : $K_{X} \rightarrow K_{Y}$. Then the compatibility with the differential of $L$ is clear, if it is defined in $\mathcal{H o m}_{\text {Diff }}^{f}$. For the proof of the isomorphism we may assume that $L$ is a coherent $\mathcal{O}_{X}$-Module. Then (0.5) is quasi-isomorphic to the composition :

$$
\begin{aligned}
f_{\bullet} \mathcal{H o m}_{\mathcal{O}_{X}}\left(L, K_{X}\right) & \longrightarrow \mathcal{H o m}_{\mathcal{O}_{Y}}\left(\mathbb{R} f_{\bullet} L, f_{\bullet} K_{X}\right) \\
& \longrightarrow \mathcal{H o m}_{\mathcal{O}_{Y}}\left(\mathbb{R} f_{\bullet} L, K_{Y}\right)
\end{aligned}
$$

and the assertion is reduced to the dualities in $[\mathrm{H}]$, [RRV]. Here $\mathbb{R} f_{\bullet} L$ is defined by choosing some canonical $f_{\bullet}$-acyclic resolution of $L$.

We also show the compatibility of the dual functor with the de Rham functor $D R \mathbb{D}=\mathbb{D} D R$ in the holonomic case using the forgetful functor of the differential complexes, where the proof of the isomorphism is same as in [K1]. Then the compatibility of the duality for proper morphisms (0.1) with the topological (i.e. Verdier) duality by the de Rham functor becomes trivial, because $\operatorname{Tr}_{f}: \widetilde{K}_{X} \rightarrow \widetilde{K}_{Y}$ represents the topological trace morphism $\operatorname{Tr}_{f}: \mathbb{R} f_{\bullet} \mathbb{C}_{X}\left[2 d_{X}\right] \rightarrow \mathbb{C}_{Y}\left[2 d_{Y}\right]$.

These results were first proved in the filtered case in [S1, paragraph 2], where the arguments are sometimes simplified (e.g. the stability of the coherence by the direct image by proper morphisms) due to the existence of the global filtration. In fact, we have to use the filtrered theory to relate the two trace morphisms $\operatorname{Tr}_{f}: \widetilde{K}_{X} \rightarrow \widetilde{K}_{Y}$ and $\operatorname{Tr}_{f}: K_{X} \rightarrow K_{Y}$ (see 3.7).

Here it should be noted that

$$
F_{p} \mathcal{H o m}_{\text {Diff }}\left(L, L^{\prime}\right):=\mathcal{H}_{o} m_{\mathcal{O}_{X}}\left(L, L^{\prime} \otimes F_{p} \mathcal{D}_{X}\right)
$$

the sheaf of the differential morphisms of order $\leq p$ coincides with $\mathcal{D i f f}_{X}^{p}\left(L, L^{\prime}\right)$ the differential operators of order $\leq p$ in the sense of GROTHENDIECK [BO] (see (1.20.2)).

We also introduce the notion of diagonal pairing to simplify some argument in the proof of the fully faithfulness of the Riemann-Hilbert 
correspondence, $c f$. 4.7. This can be also used to define the duality isomorphism, $c f .4 .8$, and might simplify some arguments which should be needed in the proof of [Sc 1-2] (see 4.9).

In paragraph 1 we define the induced $\mathcal{D}$-Modules and the differential complexes, and prove some equivalence of categories to assure the existence of some resolution. In paragraph 2 we define the dual and prove the compatibility with the de Rham functor in the holonomic case. In paragraph 3 we show the duality for proper morphisms and its compatibility with the topological one. In paragraph 4 we explain about the diagonal pairings.

I would like to thank Professors A. Borel, P. Deligne, M. Kashiwara and B. Malgrange for useful discussions and good suggestions. This research was partially supported by NSF grant DMS 8610730 .

\section{Induced $\mathcal{D}$-Modules and Differential Complexes}

1.0. - In this note $X$ denotes a complex manifold, or a smooth algebraic variety, and $\mathcal{D}_{X}$ the sheaf of holomorphic, or algebraic, differential operators. In the algebraic case, the $\mathcal{O}_{X^{-}}\left(\right.$and $\left.D_{X^{-}}\right)$Modules are assumed quasi-coherent (except in paragraph 4). We identify the left and right $\mathcal{D}_{X^{-}}$ Modules by the functor $M \mapsto \Omega_{X}^{d_{X}} \otimes_{\mathcal{O}_{X}} M$, where $d_{X}:=\operatorname{dim} X$. We use mainly the right $\mathcal{D}$-Modules, because they are more convenient to the definition of dual and the proof of the duality (in fact, the induced $\mathcal{D}$-Modules are naturally defined as right $\mathcal{D}$-Modules).

1.1 Definition. - A $\mathcal{D}_{X}$-Module $M$ is induced, if it is isomorphic to $L \otimes \mathcal{O}_{X} \mathcal{D}_{X}$ for an $\mathcal{O}_{X}$-Module $L . M_{i}\left(\mathcal{D}_{X}\right)$ denotes the additive category of the induced $\mathcal{D}_{X}$-Modules, which is a full sub category of the abelian category of $\mathcal{D}_{X}$-Modules $M\left(\mathcal{D}_{X}\right)$. Then $C_{i}^{b}\left(\mathcal{D}_{X}\right), K_{i}^{b}\left(\mathcal{D}_{X}\right), D_{i}^{b}\left(\mathcal{D}_{X}\right)$ and $C^{b}\left(\mathcal{D}_{X}\right)$, etc. (same for $C_{i}, C_{i}^{+}, C_{i}^{-}$, etc.) are defined as in [V1], where $D_{i}^{b}\left(\mathcal{D}_{X}\right), D^{b}\left(\mathcal{D}_{X}\right)$, etc. are obtaind by inversing the quasi-isomorphisms in $K_{i}^{b}\left(\mathcal{D}_{X}\right), K^{b}\left(\mathcal{D}_{X}\right)$, etc.

1.2 LEMMA. - For induced $\mathcal{D}_{X}$-Modules $M, N$, we have $M \otimes_{\mathcal{D}_{X}}^{\mathbb{L}} \mathcal{O}_{X}=$ $M \otimes_{\mathcal{D}_{X}} \mathcal{O}_{X}$, i.e. $\mathcal{T o r}_{i}^{\mathcal{D}_{X}}\left(M, \mathcal{O}_{X}\right)=0$ for $i \neq 0$, and the natural morphism

$$
\mathcal{H o m}_{D_{X}}(M, N) \longrightarrow \mathcal{H o m}_{\mathbb{C}_{X}}\left(M \otimes_{\mathcal{D}_{X}} \mathcal{O}_{X}, N \otimes_{\mathcal{D}_{X}} \mathcal{O}_{X}\right)
$$

is injective.

Proof. - The first assertion is clear, and the second is shown in $[S 1,2.2 .2]$.

1.3 Definition. - For $\mathcal{O}_{X}$-Modules $L, L^{\prime}$, we denote by $\mathcal{H o m}_{\text {Diff }}\left(L, L^{\prime}\right)$ TOME $117-1989-\mathrm{N}^{\circ} 3$ 
and call the differential morphisms of $L$ to $L^{\prime}$ the image of the injection (1.2.1) :

$$
\mathcal{H o m}_{\mathcal{D}_{X}}\left(L \otimes_{\mathcal{O}_{X}} \mathcal{D}_{X}, L^{\prime} \otimes_{\mathcal{O}_{X}} \mathcal{D}_{X}\right) \longrightarrow \mathcal{H o m}_{\mathbb{C}_{X}}\left(L, L^{\prime}\right)
$$

We denote by $M\left(\mathcal{O}_{X}\right.$, Diff $)$ the additive category whose objects are the $\mathcal{O}_{X}$-Modules, and morphisms are the differential morphisms. We have a natural functor

$$
\widetilde{\mathrm{DR}}^{-1}: M\left(\mathcal{O}_{X}, \mathrm{Diff}\right) \longrightarrow M_{i}\left(\mathcal{D}_{X}\right)
$$

such that $\widetilde{D R}^{-1}(L)=L \otimes \mathcal{O}_{X} \mathcal{D}_{X}$, and it is an equivalence of categories by the injectivity of (1.2.1). We define $C^{b}\left(\mathcal{O}_{X}\right.$, Diff $), K^{b}\left(\mathcal{O}_{X}\right.$, Diff $)$ as above, and $D^{b}\left(\mathcal{O}_{X}\right.$, Diff) by inversing the $D$-quasi-isomorphisms (same for $D, D^{+}$, etc.). Here a morphism of $C^{b}\left(\mathcal{O}_{X}\right.$, Diff) is called a $D$-quasiisomorphism, if its image by $\widetilde{D R}^{-1}$ is a quasi-isomorphism (similar for $D$-acyclic). By definition we have the equivalence of categories

$$
\widetilde{D R}^{-1}: D^{b}\left(\mathcal{O}_{X}, \text { Diff }\right) \stackrel{\sim}{\longrightarrow} D_{i}^{b}\left(\mathcal{D}_{X}\right)
$$

(same for $C^{b}, K^{b}$, and $D, D^{+}$, etc.).

1.4. Remark. - The morphism (1.3.1) is induced by the following commutative diagrams for $P \in \mathcal{H o m}_{\mathcal{D}_{X}}\left(L \otimes \mathcal{D}_{X}, L^{\prime} \otimes \mathcal{D}_{X}\right)=$ $\mathcal{H o m}_{\mathcal{O}_{X}}\left(L, L^{\prime} \otimes \mathcal{D}_{X}\right):$
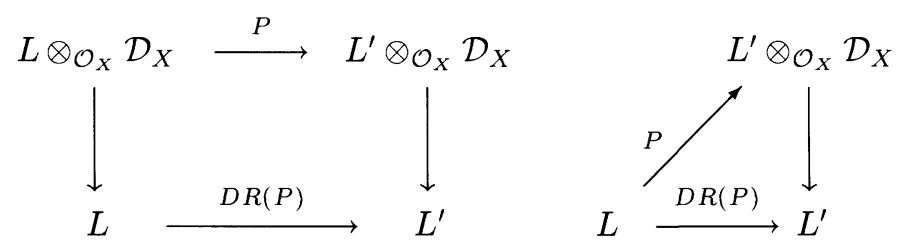

where $D R(P)$ is the image of $P$ by (1.3.1), and the vertical morphisms are defined by the tensor over $\mathcal{O}_{X}$ of the natural morphism $\mathcal{D}_{X} \rightarrow \mathcal{O}_{X}$ $(Q \mapsto Q 1)$.

1.5. Lemma. - For $M \in M\left(\mathcal{D}_{X}\right)$, put

$$
\begin{aligned}
\widetilde{D R}(M):=\left[0 \longrightarrow M \otimes_{\mathcal{O}_{X}} \Lambda^{d_{X}} \Theta_{X} \longrightarrow \cdots\right. \\
\left.\cdots \longrightarrow M \otimes_{\mathcal{O}_{X}} \Theta_{X} \longrightarrow M \longrightarrow 0\right]
\end{aligned}
$$

BULLETIN DE LA SOCIÉTÉ MATHÉMATIQUE DE FRANCE 
where $\Theta_{X}$ is the sheaf of holomorphic or algebraic vector fields, and the degree of $M$ is zero. Choosing coordinates $\left(x_{1}, \ldots, x_{d_{X}}\right)$ and their dual vector fields $\partial_{1}, \ldots, \partial_{d_{X}},(1.5 .1)$ is identified with the Koszul complex $K\left(M ; \partial_{1}, \ldots, \partial_{d_{X}}\right)\left[d_{X}\right],(c f .[\mathrm{KK}]$ for the intrinsic definition). Then $\widetilde{D R}(M)$ is a complex of $M\left(\mathcal{O}_{X}\right.$, Diff $)$ by the natural $\mathcal{O}_{X}$-Module structure on $M \otimes \Lambda^{i} \Theta_{X}$, and we have a natural quasi-isomorphism of complexes of $\mathcal{D}_{X}$-Modules

$$
\widetilde{D R}^{-1} \widetilde{D R}(M) \longrightarrow M
$$

where $\widetilde{D R}^{-1} M=M \otimes \mathcal{D}_{X} \rightarrow M$ is induced by $\mathcal{D}_{X} \rightarrow \mathcal{O}_{X}$ as in 1.4.

Proof. - This is a special case of $[\mathrm{S} 1,2.1 .6,2.2 .8]$, where $F$ on $M$ is trivial.

1.6. Remark. - $\widetilde{D R}^{-1} M=M \otimes_{\mathcal{O}_{X}} \mathcal{D}_{X}$ has two structures of right $\mathcal{D}_{X}$-Modules which are interchangeable by the lemma below. We have a natural isomorphism of $\widetilde{D R}\left(\widetilde{D R}^{-1} M\right)$ with $\widetilde{D R}^{-1} \widetilde{D R}(M)$, and $\widetilde{D R}\left(\widetilde{D R}^{-1} M\right)$ is just the Koszul complex $K\left(M \otimes_{\mathcal{O}_{X}} \mathcal{D}_{X} ; \partial_{1}, \ldots, \partial_{d_{X}}\right)\left[d_{X}\right]$.

1.7. Lemma. - For a right $\mathcal{D}_{X}$-Module $M$, there is a unique involution of $M \otimes_{\mathcal{O}_{X}} \mathcal{D}_{X}$, which induces the identity on $M=M \otimes 1$, and exchanges the two structures of right $\mathcal{D}_{X}$-Module on $M \otimes_{\mathcal{O}_{X}} \mathcal{D}_{X}$ : one is associated with the tensor over $\mathcal{O}_{X}$, and the other is the right multiplication of $\mathcal{D}_{X}$.

Proof. - We denote by $t(P)$ the action of $P \in \mathcal{D}_{X}$ associated with the tensor over $\mathcal{O}_{X}$ of right and left $\mathcal{D}_{X}$-Modules, i.e. $(m \otimes n) t(a)=m a \otimes n$, $(m \otimes n) t(v)=m v \otimes n-m \otimes v n$ for $a \in \mathcal{O}_{X}, v \in \Theta_{X}$. Then the above involution $\iota$ must satisfy $\iota(m \otimes P)=(m \otimes 1) t(P)$, and we can easily check $\iota^{2}=$ id using local coordinates, which completes the proof ( $c f$. [S1, 2.4.2]).

Remark. - If we fix the coordinates $\left(x_{1}, \ldots, x_{d_{X}}\right)$, and identify $\omega_{X} \otimes \mathcal{O}_{X} \mathcal{D}_{X}$ with $\mathcal{D}_{X}$ by $d x \otimes P \leftrightarrow P$, then the involution of $\omega_{X} \otimes \mathcal{D}_{X}$ is identified with the involution $*$ of $\mathcal{D}_{X}$ defined by $(P Q)^{*}=Q^{*} P^{*}, x_{i}^{*}=x_{i}$, $\partial_{i}^{*}=-\partial_{i}^{\prime}$. This involution is essentially used in the proof of $M^{\bullet} \stackrel{\sim}{\rightarrow} \mathbb{D D D} M^{\bullet}$ for $M^{\bullet} \in D_{\text {coh }}^{b}\left(\mathcal{D}_{X}\right)$.

1.8. Proposition. - We have an equivalence of categories

$$
\widetilde{D R}^{-1}: D^{b}\left(\mathcal{O}_{X}, \text { Diff }\right) \stackrel{\sim}{\longrightarrow} D_{i}^{b}\left(\mathcal{D}_{X}\right) \stackrel{\sim}{\longrightarrow} D^{b}\left(\mathcal{D}_{X}\right)
$$

where a quasi-inverse is given by $\widetilde{D R}$ (same for $D, D^{+}, D^{-}$).

Proof. - By 1.5 and [V1], $D_{i}^{b}\left(\mathcal{D}_{X}\right) \rightarrow D^{b}\left(\mathcal{D}_{X}\right)$ is an equivalence of categories, and $\widetilde{D R}^{-1} \widetilde{D R}$ is isomorphisc to id.

TOME $117-1989-\mathrm{N}^{\circ} 3$ 
1.9. LemmA. - For $L \in M\left(\mathcal{O}_{X}\right.$, Diff $)$, we have a functorial quasiisomorphism

$$
\widetilde{D R}\left(\widetilde{D R}^{-1} L\right) \longrightarrow L \quad \text { in } C^{b}\left(\mathcal{O}_{X}, \text { Diff }\right)
$$

where $\widetilde{D R}^{-1} L=L \otimes \mathcal{D}_{X} \rightarrow L$ is induced by $\mathcal{D}_{X} \rightarrow \mathcal{O}_{X}$ as in 1.5 .

Proof. - The last morphism $L \otimes \mathcal{D}_{X} \rightarrow L$ corresponds to the morphism $L \otimes \mathcal{D}_{X} \otimes_{I_{X}} \mathcal{D}_{X} \rightarrow L \otimes \mathcal{D}_{X}$ defined by $u \otimes P \otimes Q \rightarrow u \otimes P Q$, and is defined in $M\left(\mathcal{O}_{X}\right.$, Diff $)$. Then the assertion is easily checked using the Koszul complex $K\left(L \otimes \mathcal{D}_{X} ; \partial_{1}, \ldots, \partial_{d_{X}}\right)$.

Remark. - $\widetilde{D R}^{-1}$ of (1.9.1) is isomorphic to (1.5.2) applied to $M=L \otimes \mathcal{D}_{X}$ using the involution 1.7.

1.10. Corollary. - $L^{\bullet} \in C\left(\mathcal{O}_{X}\right.$, Diff $)$ is acyclic, if it is $D$-acyclic, (cf. 1.3), and a morphism of $C\left(\mathcal{O}_{X}\right.$, Diff $)$ is an quasi-isomorphism, if it is a D-quasi-isomorphism.

1.11 Definition. - We define subsheaves of $\mathcal{H o m}_{\text {Diff }}\left(L, L^{\prime}\right)=$ $\mathcal{H o m}_{\mathcal{D}_{X}}\left(L \otimes \mathcal{D}_{X}, L^{\prime} \otimes \mathcal{D}_{X}\right)$ by

$$
\begin{gathered}
F_{p} \mathcal{H o m}_{\mathrm{Diff}}\left(L, L^{\prime}\right)=F_{p} \mathcal{H o m}_{\mathcal{D}_{X}}\left(L \otimes \mathcal{D}_{X}, L^{\prime} \otimes \mathcal{D}_{X}\right) \\
:=\mathcal{H o m}_{I_{X}}\left(L, L^{\prime} \otimes F_{p} \mathcal{D}_{X}\right) \hookrightarrow \mathcal{H o m}_{I_{X}}\left(L, L^{\prime} \otimes \mathcal{D}_{X}\right) \\
\quad=\mathcal{H o m}_{\mathcal{D}_{X}}\left(L \otimes \mathcal{D}_{X}, L^{\prime} \otimes \mathcal{D}_{X}\right)=\mathcal{H o m}_{\text {Diff }}\left(L, L^{\prime}\right)
\end{gathered}
$$

and put

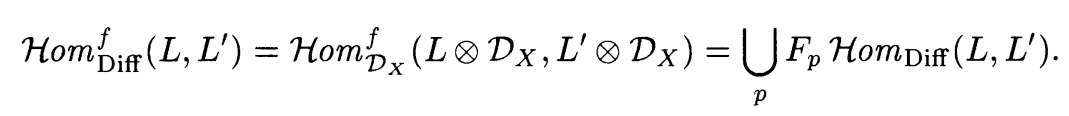

A differential morphism belonging to $\mathcal{H o m}_{\text {Diff }}^{f}\left(L, L^{\prime}\right)$ (resp. to $\left.F_{p} \mathcal{H o m}_{\text {Diff }}\left(L, L^{\prime}\right)\right)$ is called of finite order (resp. of order $\leq p$ ). Let $M\left(\mathcal{O}_{X}, \text { Diff }\right)^{f}$ be the subcategory of $M\left(\mathcal{O}_{X}\right.$, Diff $)$ whose morphisms are the differential morphisms of finite orders, and define $C^{b}\left(\mathcal{O}_{X} \text {, Diff }\right)^{f}$, $D^{b}\left(\mathcal{O}_{X}, \text { Diff }\right)^{f}$, etc. as in 1.3.

Remarks.

i) $\mathcal{H o m}_{\text {Diff }}^{f}\left(L, L^{\prime}\right)=\mathcal{H o m}_{\text {Diff }}\left(L, L^{\prime}\right)$, if $L$ is coherent.

ii) For a complex of $\mathcal{D}_{X^{-}}$-Modules $M^{\bullet}, \widetilde{D R}\left(M^{\bullet}\right)$ is a complex of $M\left(\mathcal{O}_{X} \text {, Diff }\right)^{f}$, because the differential of $M^{\bullet}$ is $\mathcal{O}_{X}$-linear, i.e. order 0 , and that of $\widetilde{\mathrm{DR}}\left(M^{j}\right)$ is order 1 .

iii) $\mathcal{H o m}_{\mathcal{D}_{X}}^{f}\left(M, M^{\prime}\right)$ is not well-defined for induced $\mathcal{D}$-Modules $M, M^{\prime}$ unless the isomorphisms $M=L \otimes \mathcal{D}_{X}$, etc. are chosen. 
1.2 Proposition. - We have an equivalence of categories

$$
\widetilde{D R}^{-1}: D^{b}\left(\mathcal{O}_{X}, \text { Diff }\right)^{f} \stackrel{\sim}{\longrightarrow} D^{b}\left(\mathcal{D}_{X}\right)
$$

with a quasi-inverse $\widetilde{D R}$.

Proof. - By 1.5, $\widetilde{D R}^{-1} \circ \widetilde{D R} \simeq \mathrm{id}$, and it is enough to construct a relatively functorial isomorphism $\widetilde{D R}\left(\widetilde{D R}^{-1} L\right) \simeq L$ in $D^{b}\left(\mathcal{O}_{X}, \text { Diff }\right)^{f}$. With the notations of the two lemmas below, we have $D$-quasi-isomorphisms in $C^{b}\left(\mathcal{O}_{X}, \text { Diff }\right)^{f}$ :

$$
\widetilde{D R}\left(\widetilde{D R}^{-1} L\right) \longleftarrow F_{p} \widetilde{D R}\left(\widetilde{D R}^{-1}\right) \longrightarrow L
$$

for $p \geq 0$, if we define $F$ on $M=L \otimes_{\mathcal{O}_{X}} \mathcal{D}_{X}$ as in 1.14 below. In fact $L \otimes \mathcal{D}_{X} \rightarrow L$ in 1.9. has infinite order, because it corresponds to id $\in \mathcal{H o m}_{\mathcal{O}_{X}}\left(L \otimes \mathcal{D}_{X}, L \otimes \mathcal{D}_{X}\right)$, but its restriction to $L \otimes F_{p} \mathcal{D}_{X}$ has finite order $p$. Then in the algebraic case, a differential morphism of finite order preserves the filtration $F$ of $\widetilde{D R}\left(\widetilde{D R}^{-1} L\right)$ up to shift globally, and we get the assertion. In the analytic case, for a finite number of $\mathcal{O}_{X^{-}}$ Modules and differential morphisms of finite orders between them such that the directions of the morphisms are compatible with some ordering of the $\mathcal{O}_{X}$-Modules, we have a locally finite covering $\left\{U_{i}\right\}$ of $X$ such that the morphisms preserves the filtration $F$ of $\widetilde{D R}\left(\widetilde{D R}^{-1} L\right)$ up to shift on $U_{I}:=\bigcap_{i \in I} U_{i}$ for any $I$. Moreover we can choose the shift so that the natural inclusion $\left(j_{I}\right) ! j_{I}^{-1} \rightarrow\left(j_{I^{\prime}}\right) ! j_{I^{\prime}}^{-1}$ for $I \supset I^{\prime}$ preserves the filtration, where $j_{I}: U_{I} \rightarrow X$. Then we replace $(1.12 .1)$ by the co-Čech complex using $\left(j_{I}\right)_{!} j_{I}^{-1}$, and we get the assertion.

1.13 Lemma. - For a filtered $\mathcal{D}_{X}$-Module $(M, F)$, i.e. $\left(F_{p} M\right) F_{q} \mathcal{D}_{X} \subset$ $F_{p+q} M, \cup F_{p} M=M$ and $F_{p} M=0$ for $p<<0$ locally on $X$, let $F, \widetilde{F}$ be the filtration of $\widetilde{D R}(M), \widetilde{D R}^{-1} \widetilde{D R}(M)=\widetilde{D R}\left(M \otimes \mathcal{D}_{X}\right)$ (cf. 1.6.) such that $F_{p}\left(M \otimes \Lambda^{i} \Theta_{X}\right)=F_{p-i} M \otimes \Lambda^{i} \Theta_{X}, \widetilde{F}_{p}\left(\left(M \otimes \mathcal{D}_{X}\right) \otimes_{\mathcal{O}_{X}} \Lambda^{i} \Theta_{X}\right)=$ $\left(F_{p-i} M \otimes \mathcal{O}_{X} \mathcal{D}_{X}\right) \otimes \mathcal{O}_{X} \Lambda^{i} \Theta_{X}$. Then $F, \widetilde{F}$ are filtrations in $C^{b}\left(\mathcal{O}_{X}\right.$, Diff $)$, $C_{i}^{b}\left(\mathcal{D}_{X}\right)$ such that $\operatorname{Gr}_{p}^{F} \widetilde{D R}(M)$ are complexes of $\mathcal{O}_{X}$-Modules and

$$
\begin{aligned}
\widetilde{F}_{p} \widetilde{D R}\left(M \otimes \mathcal{D}_{X}\right) & =\widetilde{D R}^{-1}\left(F_{p} \widetilde{D R}(M)\right), \\
\operatorname{Gr}_{p} \widetilde{F} \widetilde{D R}\left(M \otimes \mathcal{D}_{X}\right) & =\left(\operatorname{Gr}_{p}^{F} \widetilde{D R}(M)\right) \otimes_{\boldsymbol{I}_{X}} \mathcal{D}_{X} .
\end{aligned}
$$

Proof. - The assertion is clear by definition (cf. 1.6).

TOME $117-1989-\mathrm{N}^{\circ} 3$ 
1.14. LEMMA. - With the notation as above, $\operatorname{Gr}_{p}^{F} \widetilde{D R}(M)$ is acyclic for $p>0$ (resp. $p>>0$ locally on $X$ ), if $(M, F)=L \otimes_{\mathcal{O}_{X}}\left(\mathcal{D}_{X}, F\right)$, i.e. $F_{p} M=L \otimes_{\mathcal{O}_{X}} F_{p} \mathcal{D}_{X}$ (resp. $M$ is coherent and $F$ is a good filtration, i.e. $\operatorname{Gr}^{F} M$ is coherent over $\left.\operatorname{Gr}^{F} \mathcal{D}_{X}\right)$. If $\operatorname{Gr}_{p}^{F} \widehat{D R}(M)$ are acyclic for $p>p_{0}$, the natural morphisms

$$
\widetilde{D R}^{-1} \widetilde{D R}(M) \longleftarrow \widetilde{D R}^{-1} F_{p} \widetilde{D R}(M) \longrightarrow M
$$

are quasi-isomorphisms for $p \geq p_{0}$.

Proof. - The first assertion is clear for $M=L \otimes \mathcal{D}_{X}$, because $\mathrm{Gr}^{F} \widehat{D R}(M)$ is the Koszul complex

$$
K\left(L \otimes \mathcal{O}_{X} \mathcal{O}_{X}\left[\xi_{1}, \ldots, \xi_{d_{X}}\right] ; \xi_{1}, \ldots, \xi_{d_{X}}\right)\left[d_{X}\right]
$$

with $\xi_{i}=\operatorname{Gr} \partial_{i}$.

The coherent case is then reduced to this case using a filtered resolution of $(M, F)$ by filtered free $\mathcal{D}_{X}$-Modules of finite rank, which exists locally (cf. [S1, 2.1.17]). (Here we do not need the finite length of the resolution and Cartan's theorem $A$ is enough). The last assertion is clear by 1.5 and 1.13 .

1.15 Definition. - $D_{\text {coh }}\left(\mathcal{D}_{X}\right)$ (resp. $\left.D_{\text {hol }}\left(\mathcal{D}_{X}\right)\right)$ is the full subcategory of $D\left(\mathcal{D}_{X}\right)$ defined by the condition : $\mathcal{H}^{j} M^{\bullet}$ are coherent (resp. holonomic) (same for $D_{\text {coh }}^{b}, D_{\text {coh }}^{+}$, etc.). We define $D_{\text {coh }}^{b}\left(\mathcal{O}_{X}, \text { Diff }\right)^{f}$, etc. naturally so that

$$
\widetilde{D R}^{-1}: D_{\mathrm{coh}}^{b}\left(\mathcal{O}_{X}, \text { Diff }\right)^{f} \stackrel{\sim}{\longrightarrow} D_{i, \mathrm{coh}}^{b}\left(\mathcal{D}_{X}\right) \stackrel{\sim}{\longrightarrow} D_{\mathrm{coh}}^{b}\left(\mathcal{D}_{X}\right)
$$

(same for hol). A complex in $D_{\text {coh }}^{b}\left(\mathcal{O}_{X}\right.$, Diff) (resp. $D_{\text {hol }}^{b}\left(\mathcal{O}_{X}\right.$, Diff)) is called $D$-coherent (resp. $D$-holonomic). We say that $M$ is a good coherent $\mathcal{D}_{X}$-Module, if for any relatively compact open subset $U$ of $X$, there exists a finite filtration $G$ of $\left.M\right|_{U}$ of $\mathcal{D}_{X}$-Modules such that $\left.\operatorname{Gr}_{p}^{G} M\right|_{U}$ are coherent $\mathcal{D}_{U}$-Modules with good filtrations. In the algebraic case this notion is same as the coherence $(c f$. $[\mathrm{B}, \mathrm{Be}])$. Let $D_{g, \text { coh }}^{b}\left(\mathcal{D}_{X}\right)$ be the full subcategory of $D^{b}\left(\mathcal{D}_{X}\right)$ with good coherent cohomologies, and $D_{g, \text { coh }}^{b}\left(\mathcal{O}_{X}, \text { Diff }\right)^{f}$ the full subcategory of $D^{b}\left(\mathcal{O}_{X}, \text { Diff }\right)^{f}$ defined by the condition : $\widetilde{D R}^{-1} L^{\bullet} \in D_{g, \text { coh }}^{b}\left(\mathcal{D}_{X}\right)$. Put $D_{g, \text { hol }}^{b}=D_{g, \text { coh }}^{b} \cap D_{\text {hol }}$. Then

$$
\widetilde{D R}^{-1}: D_{g, \text { coh }}^{b}\left(\mathcal{O}_{X}, \text { Diff }\right)^{f} \stackrel{\sim}{\longrightarrow} D_{g, \text { coh }}^{b}\left(\mathcal{D}_{X}\right)
$$

(same for $\left.D_{g, \text { hol }}^{b}\right)(c f .1 .12)$. 
The following lemmas will be used for the definition of dual in paragraph 2 .

1.16. Lemma. - Let $L, L^{\prime}$ and $L^{\prime \prime}$ be $\mathcal{O}_{X}$-Modules. Then $u$ in $\mathcal{H o m}_{\text {Diff }}^{f}\left(L^{\prime \prime}, L\right) \quad$ and $\quad v \quad$ in $\mathcal{H o m}_{\text {Diff }}^{f}\left(L^{\prime}, L^{\prime \prime}\right)$ induce morphisms of $M\left(\mathcal{O}_{X}, \text { Diff }\right)^{f}$ :

$$
\begin{aligned}
& u^{*}: \mathcal{H o m}_{\text {Diff }}^{f}\left(L, L^{\prime}\right) \longrightarrow \mathcal{H o m}_{\text {Diff }}^{f}\left(L^{\prime \prime}, L^{\prime}\right), \\
& v_{*}: \mathcal{H o m}_{\text {Diff }}^{f}\left(L, L^{\prime}\right) \longrightarrow \mathcal{H o m}_{\text {Diff }}^{f}\left(L, L^{\prime \prime}\right)
\end{aligned}
$$

by composition, where the $\mathcal{O}_{X}$-Module structure of $\mathcal{H o m}_{\text {Diff }}^{f}\left(L, L^{\prime}\right)$, etc. is given by the composition of the action of $\mathcal{O}_{X}$ (viewed as differential morphisms of order 0 ) on the target of the morphisms $L^{\prime}$, etc.

Proof. - The assertion for $u^{*}$ is clear, because it is $\mathcal{O}_{X}$-linear (i.e. of order 0 ) by definition of the $\mathcal{O}_{X}$-structure on $\mathcal{H o m}_{\text {Diff }}^{f}$. For $v_{*}$ we use the $\mathcal{O}_{X}$-Module structure of $L^{\prime} \otimes_{\mathcal{I}_{X}} \mathcal{D}_{X}$ associated to $\otimes_{\mathcal{O}_{X}}$, and

$$
\begin{aligned}
\left(L^{\prime} \otimes_{\boldsymbol{I}_{X}} \mathcal{D}_{X}\right) \otimes_{\boldsymbol{I}_{X}} \mathcal{D}_{X} & =L^{\prime} \otimes_{\boldsymbol{I}_{X}}\left(\mathcal{D}_{X} \otimes_{\boldsymbol{I}_{X}} \mathcal{D}_{X}\right) \\
& =\left(L^{\prime} \otimes_{\boldsymbol{I}_{X}} \mathcal{D}_{X}\right) \otimes_{\mathcal{D}_{X}}\left(\mathcal{D}_{X} \otimes_{\mathcal{O}_{X}} \mathcal{D}_{X}\right)
\end{aligned}
$$

we define $v^{\prime}:\left(L^{\prime} \otimes_{1_{X}} \mathcal{D}_{X}\right) \otimes_{1_{X}} \mathcal{D}_{X} \rightarrow\left(L^{\prime \prime} \otimes_{1_{X}} \mathcal{D}_{X}\right) \otimes_{1_{X}} \mathcal{D}_{X}$ such that $D R\left(v^{\prime}\right)=v$, where $\mathcal{D}_{X} \otimes_{\mathcal{O}_{X}} \mathcal{D}_{X}$ is the tensor of left $\mathcal{D}_{X}$-Modules over $\mathcal{O}_{X}$ (cf. [S1, 2.4.8-9] for details).

Remark. - The reason why we use the $\mathcal{O}_{X}$-Module structure on $\mathcal{H o m}_{\text {Diff }}^{f}$ induced by that on the target of the morphisms is that this structure is related to the definition of dual by the next :

1.17. LEMMA. - With the notation as above, assume $L^{\prime}$ is a right $\mathcal{D}_{X}$ Module. Then by the involution 1.7 we have the canonical isomorphism

$$
\begin{aligned}
\mathcal{H o m}_{\text {Diff }}^{f}\left(L, L^{\prime}\right) & =\mathcal{H o m}_{\mathcal{D}_{X}}^{f}\left(L \otimes \mathcal{D}_{X}, L^{\prime} \otimes \mathcal{D}_{X}\right) \\
& =\mathcal{H o m}_{\boldsymbol{\prime}_{X}}\left(L, L^{\prime}\right) \otimes_{\mathcal{O}_{X}} \mathcal{D}_{X}
\end{aligned}
$$

as right $\mathcal{D}_{X}$-Modules, where the $\mathcal{D}_{X}$-Module structure on the first term is defined by the compisition with the action of $\mathcal{D}_{X}$ on $L^{\prime}$. Moreover we have a natural isomorphism as complexes of $M\left(\mathcal{O}_{X}, \mathrm{Diff}^{f}\right.$ :

$$
\widetilde{D R}\left(\mathcal{H o m}_{\mathrm{Diff}}^{f}\left(L, L^{\prime}\right)\right)=\mathcal{H o m}_{\text {Diff }}^{f}\left(L, \widetilde{D R}\left(L^{\prime}\right)\right)
$$

compatible with the morphism $u^{*}, v_{*}$ in 1.16 , where $v$ is assumed $\mathcal{D}_{X^{-}}$ linear.

$$
\text { TOME } 117-1989-\mathrm{N}^{\mathrm{o}} 3
$$


Proof. - The first assertion follows from the definition of the right $\mathcal{D}_{X}$-Module structures, and the last from 1.6.

1.18. Remark. - For $u \in F_{p} \mathcal{H o m}_{\text {Diff }}\left(L, L^{\prime}\right)=\mathcal{H o m}_{\mathcal{O}_{X}}\left(L, L^{\prime} \otimes F_{p} \mathcal{D}_{X}\right)$ (cf. 1.11) we define its symbol $\operatorname{Gr}_{p}^{F} u \in \mathcal{H o m}_{\mathcal{O}_{X}}\left(L, L^{\prime} \otimes \operatorname{Gr}_{p}^{F} \mathcal{D}_{X}\right)$ by its composition with the natural projection. The symbol morphism

$$
\begin{aligned}
\operatorname{Gr}_{p}^{F}: F_{p} \mathcal{H o m}_{\text {Diff }}\left(L, L^{\prime}\right) \longrightarrow & \mathcal{H o m}_{\mathcal{O}_{X}}\left(L, L^{\prime} \otimes \operatorname{Gr}_{p}^{F} \mathcal{D}_{X}\right) \\
& =\mathcal{H o m}_{\mathcal{O}_{X}}\left(L, L^{\prime}\right) \otimes_{\mathcal{O}_{X}} \operatorname{Gr}_{p}^{F} \mathcal{D}_{X}
\end{aligned}
$$

is not surjective in general, because the filtration $F$ on $L^{\prime} \otimes \mathcal{D}_{X}$ does not splits as right $\mathcal{O}_{X}$-Modules even locally, e.g. $L^{\prime}=\mathbb{C}\{x\} /\left(x^{2}\right)$. But if $L$ or $L^{\prime}$ has a connection or a structure of right $\mathcal{D}$-Module, the symbol morphism (1.18.1) is surjective using the following

1.19 Remark. - For $u \in F_{p} \mathcal{H}_{\text {om }}$ Diff $\left(L, L^{\prime}\right)$ and $\nu=\left(\nu_{1}, \ldots, \nu_{d_{X}}\right) \in$ $\mathbb{N}^{d_{X}}$ such that $|\nu|:=\sum \nu_{i}=p$, define

$$
u_{\nu}=(-1)^{p} \prod_{i}\left(\left(\nu_{i} !\right)^{-1}\left(\operatorname{ad} x_{i}\right)^{\nu_{i}}\right) u \in \mathcal{H o m}_{\mathcal{O}_{X}}\left(L, L^{\prime}\right)
$$

where $\left(x_{1}, \ldots, x_{d_{X}}\right)$ are local coordinates. Here $(\operatorname{ad} g) u:=g \circ u-u \circ$ $g \in F_{p-1} \mathcal{H o m}_{\text {Diff }}\left(L, L^{\prime}\right)$ for $g \in \mathcal{O}_{X}, u \in F_{p} \mathcal{H o m}_{\text {Diff }}\left(L, L^{\prime}\right)$, because $\operatorname{Gr}_{p}^{F}((\operatorname{ad} g) u)=0$. Then we have

$$
\operatorname{Gr}_{p}^{F} u=\sum u_{\nu} \otimes \xi^{\nu}
$$

where $\xi_{i}=\operatorname{Gr}^{F} \partial_{i}$. In fact we can first reduce to the case $L=\mathcal{O}_{X}$ by considering any $\mathcal{O}_{X}$-linear morphisms $\mathcal{O}_{X} \rightarrow L$ (locally defined), because (1.19.1) is compatible with the composition with $\mathcal{O}_{X}$-linear morphisms. Then we may assume $u=u^{\prime} P$ with $u^{\prime} \in \mathcal{H o m}_{\mathcal{O}_{X}}\left(\mathcal{O}_{X}, L^{\prime}\right), P \in F_{p} \mathcal{D}_{X}=$ $F_{p} \mathcal{H o m}_{\text {Diff }}\left(\mathcal{O}_{X}, \mathcal{O}_{X}\right)$, because $\mathcal{H o m}_{\mathcal{O}_{X}}\left(\mathcal{O}_{X}, L^{\prime}\right) \otimes \operatorname{Gr}_{p}^{F} \mathcal{D}_{X}$ is generated by such $u=u^{\prime} P$. Therefore the assertion is reduced to the case $L=L^{\prime}=\mathcal{O}_{X}$, and it is clear.

Here note also that $\operatorname{Gr}_{p+q}^{F}(v u)=\operatorname{Gr}_{q}^{F} v \circ \operatorname{Gr}_{p}^{F} u$ for $u$ in $F_{p} \mathcal{H o m}_{\text {Diff }}\left(L, L^{\prime}\right)$ and $v$ in $F_{q} \mathcal{H}_{0 m_{\text {Diff }}}\left(L^{\prime}, L^{\prime \prime}\right)$, where we use a natural isomorphism

$$
\begin{aligned}
& \mathcal{H o m}_{\mathcal{O}_{X}}\left(L, L^{\prime} \otimes_{\mathcal{O}_{X}} \operatorname{Gr}_{p}^{F} \mathcal{D}_{X}\right) \\
& \longrightarrow \mathcal{H o m}_{\mathrm{Gr}^{F} \mathcal{D}_{X}}\left(L \otimes_{\mathcal{O}_{X}} \operatorname{Gr}^{F} \mathcal{D}_{X}, L^{\prime} \otimes_{\mathcal{O}_{X}} \operatorname{Gr}^{F[-p]} \mathcal{D}_{X}\right)
\end{aligned}
$$

with $F[i]$ the shift of filtration defined by $F[i]_{j}=F_{j-i}$. 
1.20 Remark. - A $\mathbb{C}_{X^{-}}$-linear morphism $u: L \rightarrow L^{\prime}$ of $\mathcal{O}_{X^{-}}$ Modules $L, L^{\prime}$ is called a differential operator of order $\leq p$ in the sense of Grothendieck (cf. [BO]) and denoted by $u \in \mathcal{D i f f}_{X}^{p}\left(L, L^{\prime}\right)$, iff $u$ is the composition of $L \rightarrow P_{X}^{p} \otimes_{\mathcal{O}_{X}} L$ defined by $m \hookrightarrow 1 \otimes m$ and an $\mathcal{O}_{X}$-linear morphism $\bar{u}: P_{X}^{p} \otimes_{\mathcal{O}_{X}} L \rightarrow L^{\prime}$, where $P_{X}^{p}=\delta^{\bullet} \mathcal{O}_{X \times X} / I^{p+1}$ with $I$ the ideal of the diagonal and $\delta: X \rightarrow X \times X$ the diagonal embedding. Here $\bar{u}$ is uniquely determined by $u$, because the first inclusion is factorized by $L \rightarrow \mathcal{O}_{X} \otimes_{\mathbb{C}} L=\left(\mathcal{O}_{X} \otimes_{\mathbb{C}} \mathcal{O}_{X}\right) \otimes_{\mathcal{O}_{X}} L \longrightarrow P_{X}^{p} \otimes_{\mathcal{O}_{X}} L$. For $u \in \mathcal{D i f f}_{X}^{p}\left(L, L^{\prime}\right)$, we have

$$
\prod_{i=0}^{p}\left(\operatorname{ad} g_{i}\right) u=0 \quad \text { for any } g_{i} \in \mathcal{O}_{X},
$$

because $\bar{u}:\left(\mathcal{O}_{X} \otimes_{\mathbb{C}} \mathcal{O}_{X}\right) \otimes_{\mathcal{O}_{X}} L \rightarrow L^{\prime}$ annihilates

$$
\left(\left(\mathcal{O}_{X} \otimes_{\mathbb{C}} \mathcal{O}_{X}\right) \cap I^{p+1}\right) \otimes_{\mathcal{O}_{X}} L
$$

In the algebraic case (1.20.1) is equivalent to $u \in \mathcal{D}_{\text {iff }}^{p}\left(L, L^{\prime}\right)$, because we can replace $L, L^{\prime}$, etc. by $\Gamma(U, L)$, etc. with $U$ the affine open subsets of $X$ by the quasi-coherence (but $\delta^{\bullet} \mathcal{O}_{X \times X} \neq \mathcal{O}_{X} \otimes_{\mathbb{C}} \mathcal{O}_{X}$ even in the algebraic case). In the analytic case, $\delta^{\bullet} I^{p+1} \cap \mathcal{O}_{X} \otimes_{\mathbb{C}} \mathcal{O}_{X}$ is not generated by $\Pi\left(x_{i} \otimes 1-1 \otimes x_{i}\right)^{\nu_{i}}(|\nu|=p+1)$, where $\left(x_{1}, \ldots, x_{d_{X}}\right)$ are local coordinates, and the Weierstrass preparation theorem does not hold for $\mathcal{O}_{X} \otimes_{\mathbb{C}} \mathcal{O}_{X}$.

We have, for $\mathcal{O}_{X}$-Modules $L, L^{\prime}$,

$$
F_{p} \mathcal{H o m}_{\text {Diff }}\left(L, L^{\prime}\right)=\operatorname{Diff}_{X}^{p}\left(L, L^{\prime}\right) .
$$

By the remark below we may assume $L=\mathcal{O}_{X}$. Then

$$
\begin{aligned}
F_{p} \mathcal{H o m}_{\text {Diff }}\left(\mathcal{O}_{X}, L^{\prime}\right) & =\operatorname{Diff}_{X}^{p}\left(\mathcal{O}_{X}, L^{\prime}\right) \\
& =\sum_{|\nu| \leq p} \mathcal{H o m}_{\mathcal{O}_{X}}\left(\mathcal{O}_{X}, L^{\prime}\right) \otimes \partial^{\nu}
\end{aligned}
$$

where $\partial^{\nu}=\prod \partial_{i}^{\nu_{i}} \in F_{p} \mathcal{D}_{X}=F_{p} \mathcal{H o m}_{\mathcal{D}_{X}}\left(\mathcal{O}_{X}, \mathcal{O}_{X}\right)=\mathcal{D i f f}_{X}^{p}\left(\mathcal{O}_{X}, \mathcal{O}_{X}\right)$ for $|\nu| \leq p$. In fact we have an exact sequence

$$
\begin{aligned}
0 \longrightarrow \mathcal{D i f f}_{X}^{p-1}\left(L, L^{\prime}\right) & \longrightarrow \mathcal{D i f f}_{X}^{p}\left(L, L^{\prime}\right) \\
& \longrightarrow \mathcal{H o m}_{\mathcal{O}_{X}}\left(I^{p} / I^{p+1}, \operatorname{Hom}_{\mathcal{O}_{X}}\left(L, L^{\prime}\right)\right)
\end{aligned}
$$

where the last morphism is also called the symbol morphism, and corresponds to $u \mapsto u_{\nu}$ in 1.19 by the isomorphism

$$
I^{p} / I^{p+1}=\bigoplus_{|\nu|=p} \mathcal{O}_{X} \prod_{i}\left(x_{i} \otimes 1-1 \otimes x_{i}\right)^{\nu_{i}} .
$$

TOME $117-1989-\mathrm{N}^{\circ} 3$ 
Then the assertion is clear by definition.

1.21 Remark. - For $u \in \mathcal{H o m}_{\mathbb{C}}\left(L, L^{\prime}\right)$ and $v \in \mathcal{H}^{\prime} m_{\mathcal{O}_{X}}\left(L^{\prime \prime}, L\right)$ such that $u v$ belongs to $F_{p} \mathcal{H o m}_{\text {Diff }}\left(L^{\prime \prime}, L^{\prime}\right)$ (resp. $\left.\mathcal{D i f f}_{X}^{p}\left(L^{\prime \prime}, L^{\prime}\right)\right)$, the restriction of $u$ to $\bar{L}:=\operatorname{Im} v$ belongs to $F_{p} \mathcal{H}_{0 m_{\text {Diff }}}\left(\bar{L}, L^{\prime}\right)\left(\right.$ resp. $\left.\mathcal{D i f f}_{X}^{p}\left(\bar{L}, L^{\prime}\right)\right)$. In fact, $u v$ is uniquely lifted to $D R^{-1}(u v) \in \mathcal{H o m}_{\mathcal{O}_{X}}\left(L^{\prime \prime}, L^{\prime} \otimes D_{p} \mathcal{D}_{X}\right)$ by 1.2. so that $u v$ is the composition of $D R^{-1}(u v)$ and the natural projection (cf. 1.4). Let $w: K \rightarrow L^{\prime \prime}$ be the kernel of $v$. Then $w$ is $\mathcal{O}_{X}$-linear so that

$$
D R^{-1}(u v) w=D R^{-1}(u v w)=0
$$

by 1.2 and 1.4 . Therefore $D R^{-1}(u v)$ is factorized by the $\mathcal{O}_{X}$-linear morphism $\bar{L} \rightarrow L^{\prime} \otimes F_{p} \mathcal{D}_{X}$ whose composition with the natural projection is the restriction of $u$ to $\bar{L}$. This proves the assertion for $\mathcal{H}_{0} m_{\text {Diff }}$. The case of $\operatorname{Diff}_{X}$ is similar, because $P_{X}^{p}$ is flat over $\mathcal{O}_{X}$.

\section{Duality}

2.1 Definition. - Let $\omega_{X}$ be the analytic, or algebraic, dualizing sheaf $\Omega_{X}^{d_{X}}$, and $\omega_{X}\left[d_{X}\right] \rightarrow K_{x}^{\bullet}$ a resolution as right $\mathcal{D}_{X}$-Modules such that $K_{X}^{j}$ are injective over $\mathcal{O}_{X}$ (e.g take an injective resolution as right $\mathcal{D}_{X}$-Modules, because $\mathcal{D}_{X}$ is flat over $\left.\mathcal{O}_{X}\right)$. For $L^{\bullet} \in D_{\text {coh }}^{b}\left(\mathcal{O}_{X}, \text { Diff }\right)^{f}$, $M^{\bullet} \in D_{\text {coh }}^{b}\left(\mathcal{D}_{X}\right)$, we define the dual $\mathbb{D} L^{\bullet}, \mathbb{D} M^{\bullet}$ by

$$
\begin{aligned}
\mathbb{D} L^{\bullet} & =\mathcal{H o m}_{\mathrm{Diff}}^{f}\left(L^{\bullet}, \widetilde{D R}\left(K_{X}^{\bullet}\right)\right), \\
\mathbb{D} M^{\bullet} & =\mathcal{H o m}_{\operatorname{Diff}}^{f}\left(\widetilde{D R}\left(M^{\bullet}\right), K_{X}^{\bullet}\right)
\end{aligned}
$$

(cf. 1.16-17), so that $\widetilde{\mathbb{D} R}\left(M^{\bullet}\right)=\widetilde{D R}\left(\mathbb{D} M^{\bullet}\right)$. Then $\mathbb{D} L^{\bullet}, \mathbb{D} M^{\bullet}$ are welldefined in $D^{b}\left(\mathcal{O}_{X}, \text { Diff }\right)^{f}, D^{b}\left(\mathcal{D}_{X}\right)$, and $\mathbb{D}$ is a functor of triangulated categories

$$
\begin{aligned}
& \mathbb{D}: D_{\text {coh }}^{b}\left(\mathcal{O}_{X}, \text { Diff }\right)^{f} \longrightarrow\left(D_{\text {coh }}^{b}\left(\mathcal{O}_{X}, \text { Diff }\right)^{f}\right)^{\circ}, \\
& \mathbb{D}: D_{\text {coh }}^{b}\left(\mathcal{D}_{X}\right) \longrightarrow\left(D_{\mathrm{coh}}^{b}\left(\mathcal{D}_{X}\right)\right)^{\circ}
\end{aligned}
$$

by the following lemmas :

2.2. Lemmas. - $\mathbb{D} L^{\bullet}$ is $D$-acyclic, if so is $L^{\bullet}$, and $\mathbb{D} M^{\bullet}$ is acyclic, if $s o$ is $M^{\bullet}$.

Proof. - The second assertion follows from the acyclicity of

$$
\mathcal{H o m}_{\mathcal{D}_{X}}^{f}\left(M^{\bullet} \otimes_{\mathcal{O}_{X}} \mathcal{D}_{X}, K_{X} \otimes_{\mathcal{O}_{X}} \mathcal{D}_{X}\right)=\mathcal{H o m}_{\mathcal{O}_{X}}\left(M^{\bullet}, K_{X}\right) \otimes_{\mathcal{O}_{X}} \mathcal{D}_{X}
$$


(cf. 1.17), because $\widetilde{D R}^{-1} \widetilde{D R}\left(M^{\bullet}\right)=\widetilde{D R}\left(M^{\bullet} \otimes_{\mathcal{O}_{X}} \mathcal{D}_{X}\right)$. Then for the first assertion, it is enough to show the $D$-quasi-isomorphisms (1.12.2) in the proof of 1.12 induces $D$-quasi-isomorphims on the dual, but this follows from the next :

2.3 Lemma. - Let $(M, F)$ be a filtered $\mathcal{D}_{X}$-Module such that $\operatorname{Gr}_{p}^{F} \widetilde{D R}(M)$ is acyclic for $p>p_{0},(c f .1 .13)$. Let $K$ be a right $\mathcal{D}_{X}$-Module injective over $\mathcal{O}_{X}$. Then the natural morphism

$$
\mathcal{H o m}_{\text {Diff }}^{f}(\widetilde{D R}(M), K) \longrightarrow \mathcal{H o m}_{\text {Diff }}^{f}\left(F_{p} \widetilde{D R}(M), K\right)
$$

is a quasi-isomorphism for $p \geq p_{0}$. If moreover $(M, F)=L \otimes_{\mathcal{O}_{X}}\left(\mathcal{D}_{X}, F\right)$ as in 1.14 , the morphism induced by $F_{p} \widetilde{D R}\left(L \otimes_{\mathcal{O}_{X}} \mathcal{D}_{X}\right) \rightarrow L$ in 1.9. :

$$
\mathcal{H o m}_{\text {Diff }}^{f}(L, K) \longrightarrow \mathcal{H o m}_{\text {Diff }}^{f}\left(F_{p} \widetilde{D R}\left(L \otimes_{{ }_{X}} \mathcal{D}_{X}\right), K\right)
$$

is a quasi-isomorphism for $p \geq 0$.

Proof. - The second assertion follows from the first, because we have $F_{0} \widetilde{D R}(M)=L$ by definition. Put $K_{p}^{\bullet}=\mathcal{H o m}_{\text {Diff }}^{f}\left(F_{p} \widetilde{D R}(M), K\right)$. Then it is a projective system satisfying the Mittag-Leffler condition [G], and $K_{p}^{\bullet} \rightarrow K_{q}^{\bullet}$ is a quasi-isomorphism for $p>q>p_{0}$ by assumption, because

$$
\begin{gathered}
K_{p}^{i}=\mathcal{H o m}_{\mathcal{O}_{X}}\left(F_{p-i} M \otimes \Lambda^{i} \Theta_{X}, K\right) \otimes_{\mathcal{O}_{X}} \mathcal{D}_{X} \\
\operatorname{Ker}\left(K_{p}^{\bullet} \rightarrow K_{p-1}^{\bullet}\right)=\mathcal{H o m}_{I_{X}}\left(\operatorname{Gr}_{p}^{F} \widetilde{D R}(M), K\right) \otimes_{\mathcal{O}_{X}} \mathcal{D}_{X} .
\end{gathered}
$$

Then the assertion is clear by [loc. cit.].

2.4. Lemma. - For $M^{\bullet} \in D_{\text {coh }}^{b}\left(\mathcal{D}_{X}\right)$, the natural morphism

$$
\begin{aligned}
\mathbb{D} M^{\bullet} \longrightarrow \mathbb{R} \mathcal{H o m}_{\mathcal{D}_{X}}\left(\widetilde{D R}^{-1} \widetilde{D R}\left({ }^{\bullet}\right), \widetilde{D R}^{-1} K_{X}^{\bullet}\right) \\
\quad=\mathbb{R} \mathcal{H}^{\circ} m_{\mathcal{D}_{X}}\left(M^{\bullet}, \omega_{X}\left[d_{X}\right] \otimes_{\mathcal{O}_{X}} \mathcal{D}_{X}\right)
\end{aligned}
$$

is a quasi-isomorphism. In particular, $\mathcal{H}^{j} \mathbb{D} M^{\bullet}$ are coherent (resp. holonomic) $\mathcal{D}_{X}$-Modules and $\mathcal{H}^{j} \mathbb{D} M^{\bullet}=0$ except for $-d_{X} \leq j \leq 0$ (resp. $j=0$ ), if $M$ is a coherent (resp. holonomic) $\mathcal{D}_{X}$-Module.

Proof. - The assertion is local, and we have locally a good filtration $F$ of $M$ so that $\operatorname{Gr}_{p}^{F} \widehat{D R}(M)$ is acyclic for $p>>0$. Then the first assertion follows from 2.3 , because we can replace $M$ by its free resolution, and the second from [K2].

2.5. Remark. - By V.G. Golovin (Soviet Math. Dokl. 16 (1975, p. 854), the injective dimension of $\omega_{X}$ over $\mathcal{O}_{X}$ is $d_{X}$. 
2.6. Theorem. - Let $X$ be a complex manifold. Let

$$
\text { For : } D_{\text {hol }}^{b}\left(\mathcal{O}_{X}, \text { Diff }\right)^{f} \rightarrow D_{c}^{b}\left(\mathbb{C}_{X}\right)
$$

be the forgetful functor. Put $D R=$ For $\circ \widetilde{D R}: D_{\text {hol }}^{b}\left(\mathcal{D}_{X}\right) \rightarrow D_{c}^{b}\left(\mathbb{C}_{X}\right)$. Then for $L^{\bullet} \in D_{\text {hol }}^{b}\left(\mathcal{O}_{X}, \text { Diff }\right)^{f}$, the natural morphism

$$
\begin{aligned}
\operatorname{For}\left(\mathbb{D} L^{\bullet}\right)= & \operatorname{For}\left(\mathcal{H o m}_{\mathrm{Diff}}^{f}\left(L^{\bullet}, \widetilde{D R}\left(K_{X}^{\bullet}\right)\right)\right. \\
& \longrightarrow \mathcal{H o m}_{\mathbb{C}_{X}}\left(\operatorname{For}\left(L^{\bullet}\right), \text { For } \circ \widetilde{D R}\left(K_{X}^{\bullet}\right)\right) \\
& =\mathbb{R} \mathcal{H o m}_{\mathbb{C}_{X}}\left(\operatorname{For}\left(L^{\bullet}\right), \mathbb{C}_{X}\left[2 d_{X}\right]\right)=\mathbb{D} \operatorname{For}\left(L^{\bullet}\right)
\end{aligned}
$$

is an isomorphism in $D_{c}^{b}\left(\mathbb{C}_{X}\right)$, i.e. wet get a natural functor isomorphism

$$
\text { For } \circ \mathbb{D}=\mathbb{D} \circ \text { For }: D_{\text {hol }}^{b}\left(\mathcal{O}_{X}, \text { Diff }\right)^{f} \longrightarrow\left(D_{c}^{b}\left(\mathcal{D}_{X}\right)\right)^{\circ} \text {. }
$$

Proof. - Note that the image of the functor For is contained in $D_{c}^{b}\left(\mathbb{C}_{X}\right)$ the derived category of the bounded complexes with constructible cohomologies by [K1] and 2.4. Because the assertion is local, we can reduce to the case $\mathcal{H}^{j} \widetilde{D R}^{-1} L^{\bullet}=0$ except for one $j$, and $L^{j}$ are free $\mathcal{O}_{X}$-Modules of finite type. Then the proof is same as [S1, 2.4.12].

2.7 Corollary. - We have a natural functor isomorphism $D R \circ \mathbb{D}$ $\stackrel{\sim}{\longrightarrow} \mathbb{D} \circ D R$ compatible with (2.6.1) by $\widetilde{D R} \circ \mathbb{D} \stackrel{\sim}{\longrightarrow} \mathbb{D} \circ \widetilde{D R}$ in 2.1 .

2.8. Remark. - The above proof of 2.6. is essentially same as [K1], where the perfectness of the natural pairing

$$
i_{x}^{*} \operatorname{Sol}(M) \times i_{x}^{!} D R(M) \longrightarrow \mathbb{C}
$$

i.e. $i_{x}^{*} D R(\mathbb{D} M) \stackrel{\sim}{\longrightarrow} i_{x}^{*} \mathbb{D} D R(M)$, is proved for a holonomic $\mathcal{D}_{X}$-Module $M$. Here $\operatorname{Sol}(M):=\mathbb{R} \mathcal{H o m}_{\mathcal{D}_{X}}\left(M, \omega_{X}\left[d_{X}\right]\right)$ so that $\operatorname{Sol}(M)=D R(\mathbb{D} M)$, and $i_{x}:\{x\} \rightarrow X$. This duality is generalized to $D R(\mathbb{D} M)=\mathbb{D} D R(M)$ for $M \in D_{\text {hol }}^{b}\left(\mathcal{D}_{X}\right)$ by [KK], [M1] ( $c f$. [B], [Be] for the algebraic case).

2.9. Lemma. - Let $X$ be a smooth algebraic variety, and $X^{\text {an }}$ the associated complex manifold. Let An denote the natural functors $D^{b}\left(\mathcal{O}_{X}, \text { Diff }\right)^{f} \rightarrow D^{b}\left(\mathcal{O}_{X^{\text {an }}} \text { Diff }\right)^{f}, D^{b}\left(\mathcal{D}_{X}\right) \rightarrow D^{b}\left(\mathcal{D}_{X^{\text {an }}}\right)$ induced by $\otimes_{\mathcal{O}_{X}} \mathcal{O}_{X^{\text {an }}}$ (where the pull-back by $X^{\text {an }} \rightarrow X$ is omitted). Let $K_{X \text { an }}^{\bullet}$ be an injective resolution of $\operatorname{An}\left(K_{X}^{\bullet}\right)$ as right $\mathcal{D}_{X^{\text {an }}}-$ Modules. Then for $L^{\bullet} \in D_{\text {coh }}^{b}\left(\mathcal{O}_{X}, \text { Diff }\right)^{f}$ the composition

$$
\begin{aligned}
\operatorname{An}\left(\mathcal{H o m}_{\text {Diff }}^{f}\left(L^{\bullet}, K_{X}^{\bullet}\right)\right) & \longrightarrow \mathcal{H o m}_{\text {Diff }}^{f}\left(\operatorname{An}\left(L^{\bullet}\right), \operatorname{An}\left(K_{X}^{\bullet}\right)\right) \\
& \longrightarrow \mathcal{H o m}_{\text {Diff }}^{f}\left(\operatorname{An}\left(L^{\bullet}\right), K_{X^{\text {an }}}\right)
\end{aligned}
$$

BULLETIN DE LA SOCIÉTÉ MATHÉMATIQUE DE FRANCE 
is a quasi-isomorphism, and we have the natural functor isomorphisms

$$
\begin{gathered}
\text { An } \mathbb{D}=\mathbb{D} \text { An }: D_{\text {coh }}^{b}\left(\mathcal{O}_{X}, \text { Diff }\right)^{f} \longrightarrow\left(D_{\text {coh }}^{b}\left(\mathcal{O}_{X^{\text {an }}}, \text { Diff }\right)^{f}\right)^{\circ} \\
\text { An } \mathbb{D}=\mathbb{D} \text { An }: D_{\text {coh }}^{b}\left(\mathcal{D}_{X}\right) \longrightarrow\left(D_{\text {coh }}^{b}\left(\mathcal{D}_{X^{\text {an }}}\right)\right)^{\circ} .
\end{gathered}
$$

Proof. - By definition of dual, it is enough to show the first assertion. We may assume that $\mathcal{H}^{j} \widetilde{D R}^{-1} L^{\bullet}=0$ except for one $j$, and $L^{j}$ are free $\mathcal{O}_{X}$-Modules of finite rank. Then the assertion is clear.

2.10 Theorem. - Let $X$ be as above, and For : $D_{\text {hol }}^{b}\left(\mathcal{O}_{X}, \text { Diff }\right)^{f} \rightarrow$ $D_{c}^{b}\left(\mathbb{C}_{X^{\text {an }}}\right), D R: D_{\mathrm{hol}}^{b}\left(\mathcal{D}_{X}\right) \rightarrow D_{c}^{b}\left(\mathbb{C}_{X^{\text {an }}}\right)$ the composition of An with the functors For, $D R$ in 2.6. Then we have the natural functor isomorphisms For $\mathbb{D}=\mathbb{D}$ For, $D R \mathbb{D}=\mathbb{D} D R$.

(This is clear by 2.6-7.)

2.11 Remark. - For $L^{\bullet} \in D^{b}\left(\mathcal{O}_{X} \text {, Diff }\right)^{f}$, the dual $\mathbb{D} L^{\bullet}$ is represented by the single complex associated with $\mathcal{H o m}_{\mathcal{O}_{X}}\left(L^{\bullet}, K_{X}^{\bullet}\right)$ whose first differential is given by using the identity

$$
\mathcal{H o m}_{\mathcal{O}_{X}}\left(L^{i}, K_{X}^{j}\right)=\mathcal{H o m}_{\mathcal{D}_{X}}\left(L^{i} \otimes_{\mathcal{O}_{X}} \mathcal{D}_{X}, K_{X}^{j}\right)
$$

so that

$$
\widetilde{D R}^{-1} \mathcal{H o m}_{\mathcal{O}_{X}}\left(L^{\bullet}, K_{X}^{\bullet}\right)=\mathcal{H o m}_{\mathcal{D}_{X}}^{f}\left(L^{\bullet} \otimes \mathcal{D}_{X}, K_{X}^{\bullet} \otimes \mathcal{D}_{X}\right)
$$

If $L^{i}$ are locally free, we can replace $K_{X}^{\bullet}$ by $\omega_{X}\left[d_{X}\right]$. Then the differential of the dual is given by the transpose of that of $L$ (taking local coordinates and local trivializations of $L^{i}$ ).

2.12 Remark. - In $[\mathrm{KK}]$, the duality isomorphism $D R \mathbb{D} \stackrel{\sim}{\longrightarrow} \mathbb{D} D R$ is defined by

$(2.12 .1) \quad \operatorname{Sol}(M) \otimes_{\mathbb{C}} D R(M) \longrightarrow D R\left(\omega_{X}\left[d_{X}\right]\right)=\mathbb{C}_{X}\left[2 d_{X}\right], \quad(c f .2 .8$.

We can check that it coincides with

$$
\begin{aligned}
& D R \mathbb{D}(M)=\mathbb{R} \mathcal{H o m}_{\mathcal{D}_{X}}\left(M, \omega_{X}\left[d_{X}\right]\right) \\
& \stackrel{D R}{\longrightarrow} \mathbb{R} \mathcal{H o m}_{\mathbb{C}_{X}}\left(D R(M), D R\left(\omega_{X}\left[d_{X}\right]\right)\right)=\mathbb{D} D R(M)
\end{aligned}
$$

and with that in 2.7. In fact for $u \in \mathcal{H o m}_{\text {Diff }}(L, K)$, we have a commutative diagram by 1.4 :

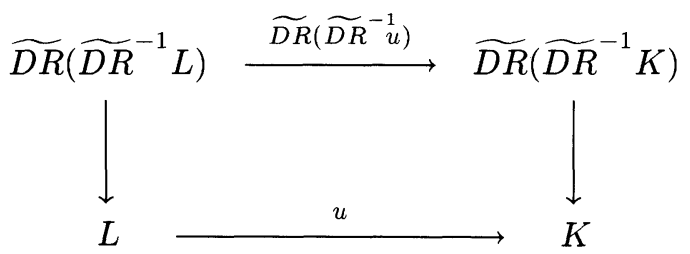

TOME $117-1989-\mathrm{N}^{\circ} 3$ 
where the vertical morphisms are as in 1.9 .

\section{Direct Images and Duality}

3.1. - Let $f: X \rightarrow Y$ be a proper morphism of complex manifolds, or smooth algebraic varieties. We denote by $f_{\bullet}$ and $f^{\bullet}$ the sheaf theoretic direct image and pull-back. The direct image of $M^{\bullet} \in D^{b}\left(\mathcal{D}_{X}\right)$ is defined by

$$
f_{*} M^{\bullet}=\mathbb{R} f_{\bullet}\left(M^{\bullet} \otimes_{\mathcal{D}_{X}}^{\mathfrak{L}} \mathcal{D}_{X \rightarrow Y}\right) \in D^{b}\left(\mathcal{D}_{X}\right),
$$

where $\mathcal{D}_{X \rightarrow Y}=\mathcal{O}_{X} \otimes_{f} \bullet \mathcal{O}_{Y} f^{\bullet} \mathcal{D}_{Y}$. To define $\otimes_{\mathcal{D}_{X}}^{\mathrm{L}}$ we take flat resolution, or factorize $f$ by $X \stackrel{i}{\rightarrow} X \times Y \stackrel{p}{\rightarrow} Y$ and use the relative de Rham $D R_{X \times Y / Y}$, where $i$ is the embedding by graph and $p$ is the projection. In this note we take the resolution of $M$ by standard induced Modules in (1.5.2). The result is same as the second definition using the canonical factorization. The merit is that we don't have to work on $X \times Y$, but only on $X, Y$. We also show that for the differential complexes the direct image is defined simply by $f_{*}$ the sheaf theoretic direct image, i.e. the differential morphisms are stable by the direct image for proper morphisms. This is obtained as a corollary of the above definition of $f_{*}$ of $\mathcal{D}_{X}$-Modules. Using this we can prove rather easily the duality for proper morphisms.

3.2. Lemma. - For an induced $\mathcal{D}_{X}$-Module $M=L \otimes_{\mathcal{O}_{X}} \mathcal{D}_{X}$, we have $\operatorname{Tor}_{i}^{\mathcal{D}_{X}}\left(M, \mathcal{D}_{X \rightarrow Y}\right)=0$ for $i \neq 0$ so that

$$
M \otimes_{\mathcal{D}_{X}}^{\mathbf{L}} \mathcal{D}_{X \rightarrow Y}=M \otimes_{\mathcal{D}_{X}} \mathcal{D}_{X \rightarrow Y}=L \otimes_{f} \bullet \mathcal{O}_{Y} f^{\bullet} \mathcal{D}_{Y} \bullet
$$

(This est clear.)

3.3. Proposition. - For $L^{\bullet} \in C^{b}\left(\mathcal{O}_{X}\right.$, Diff $)\left(\right.$ or $\left.C^{b}\left(\mathcal{O}_{X}, \text { Diff }\right)^{f}\right)$, put $M^{\bullet}=\widetilde{D R}^{-1} L^{\bullet} \in C_{i}^{b}\left(\mathcal{D}_{X}\right)$. Then $f_{\bullet} L^{\bullet} \in C^{b}\left(\mathcal{O}_{Y}\right.$, Diff) (or $\left.C^{b}\left(\mathcal{O}_{Y}, \text { Diff }\right)^{f}\right)$, i.e. the differential morphisms are stable by $f_{\bullet}$, and

$$
f_{*} M^{\bullet}=\widetilde{D R}^{-1} f_{\bullet} L^{\bullet}
$$

if $L^{i}$ are $f_{\bullet}$-acyclic.

Proof. - We consider a natural morphism $\mathcal{D}_{X \rightarrow Y} \rightarrow \mathcal{O}_{X}$ defined by the tensor of $\mathcal{D}_{X \rightarrow Y}$ with $f^{\bullet} \mathcal{D}_{Y} \rightarrow f^{\bullet} \mathcal{O}_{Y}(c f .1 .4)$ over $f^{\bullet} \mathcal{D}_{Y}$, and take the tensor of $M=L \otimes \mathcal{D}_{X}$, etc. in 1.4 with the above morphism, which induces another surjection from $L \otimes_{f^{\bullet} \mathcal{O}_{Y}} f^{\bullet} \mathcal{D}_{Y}=M \otimes_{\mathcal{D}_{X}} \mathcal{D}_{X \rightarrow Y}$, etc. in 
the first diagram of (1.4.1). Then it is enough to take the direct image of the new commutative diagram, $c f$. the remark below.

3.4. Remark. - For the reduction to the $f_{\bullet}$-acyclic case, we use an affine covering $\left\{U_{i}\right\}$ of $X$ and replace $L^{i}$ by a Čech complex $\bigoplus_{|I|=k+1}\left(j_{I}\right)_{\bullet} j_{I}^{\bullet} L^{i}$ in the algebraic case, where $j_{I}: \bigcap_{i \in I} U_{i} \rightarrow X$ and $X$ is always assumed separated. In the analytic case, we use a canonical flabby resolution of Godement (and truncate it), or a Dolbeault resolution, etc. Here $f$ is proper, and soft or fine resolution is enough. We also used the commutativity of $f_{\bullet}$ and the inductive limit in the proof of 3.3, because $\otimes_{\mathcal{O}_{Y}} \mathcal{D}_{Y}$ is the inductive limit of $\otimes_{\mathcal{O}_{Y}} F_{p} \mathcal{D}_{Y}$. As a Corollary of $3.3, f_{*} M^{\bullet}$ is well-defined in the derived category of quasi-coherent $\mathcal{D}_{Y^{-}}$ Modules in the algebraic case (compare to $[\mathrm{B}],[\mathrm{Be}]$ ).

3.5. Definition. - For $L^{\bullet} \in D^{b}\left(\mathcal{O}_{X}, \text { Diff }\right)^{f}$, we define

$$
f_{*} L^{\bullet}=\mathbb{R} f_{\bullet} L^{\bullet} \in D^{b}\left(\mathcal{O}_{X}, \text { Diff }\right)^{f}
$$

so that $f_{*} \widetilde{D R}^{-1}=\widetilde{D R}^{-1} f_{*}$, where $\mathbb{R} f_{\bullet}$ is constructed as above.

3.6. Proposition. - $D_{g, \text { coh }}^{b}\left(\mathcal{O}_{X}, \text { Diff }\right)^{f}, D_{g, \text { coh }}^{b}\left(\mathcal{D}_{X}\right)$ (cf. 1.5) are stable by the direct images for proper morphisms (same for $D_{g, \mathrm{hol}}^{b}$ ).

Proof. - By 1.14, it is enough to show that $\mathcal{H}^{j} \widetilde{D R}^{-1} f_{*} L=$ $\widetilde{D R}^{-1} \mathcal{H}^{j} \mathbb{R} f_{\bullet} L$ is a coherent $\mathcal{D}_{Y}$-Module with a good filtration for $L$ a cohernent $\mathcal{O}_{X}$-Module. But this is clear by Grauert's coherence theorem for $\mathcal{O}_{X}$-Modules.

3.7 Definition. - For a complex of filtered $\mathcal{D}_{X}$-Modules $\left(M^{\bullet}, F\right)$, we define the filtration $F$ on $\widetilde{D R}\left(M^{\bullet}\right)$ by $F_{p}\left(M^{j} \otimes \Lambda^{i} \Theta_{X}\right)=F_{p-i} M^{j} \otimes \Lambda^{i} \Theta_{X}$ so that $\widetilde{D R}\left(M^{\bullet}, F\right) \in C F\left(\mathcal{O}_{X}\right.$, Diff) (cf. [S1, 2.2]). We define $F$ on $\omega_{X}\left[d_{X}\right], K_{X}^{\bullet}$ by $\operatorname{Gr}_{p}^{F}=0$ for $p \neq 0$ so that $\omega_{X}\left[d_{X}\right] \rightarrow K_{X}^{\bullet}$ is a filtered quasi-isomorphism. The filtered trace morphism $\operatorname{Tr}_{f}$ for a proper morphism $f: X \rightarrow Y$ is a morphism $\operatorname{Tr}_{f}: \mathbb{R} f_{\bullet} \widetilde{D R}\left(\omega_{X}\left[d_{X}\right], F\right) \rightarrow$ $\widetilde{D R}\left(\omega_{Y}\left[d_{Y}\right], F\right)$ in $D F\left(\mathcal{O}_{Y}\right.$, Diff $)$ such that $\operatorname{Gr}_{0}^{F} \operatorname{Tr}_{f}$ represents the analytic, or algebraic, trace morphism $\operatorname{Tr}_{f}: \mathbb{R} f_{\bullet} \omega_{X}\left[d_{X}\right] \rightarrow \omega_{Y}\left[d_{Y}\right]$ in $D\left(\mathcal{O}_{X}\right)$ and $\operatorname{For}\left(\operatorname{Tr}_{f}\right)$ the topological one $\operatorname{Tr}_{f}: \mathbb{R} f_{\bullet} \mathbb{C}_{X}\left[2 d_{X}\right] \rightarrow \mathbb{C}_{Y}\left[2 d_{Y}\right]$, or $\operatorname{Tr}_{f}: \mathbb{R} f_{\bullet} \mathbb{C}_{X^{\text {an }}}\left[2 d_{X}\right] \rightarrow \mathbb{C}_{Y^{\text {an }}}\left[2 d_{Y}\right]$.

3.8. Remark. - If $\operatorname{Tr}_{f}$ is given in the filtered derived category as above, it can be represented by

$$
\begin{aligned}
f_{\bullet} \widetilde{D R}\left(K_{X}^{\bullet}, F\right) \longleftarrow F_{p} \widetilde{D R} & \left(\widetilde{D R}^{-1} f_{\bullet} \widetilde{D R}\left(K_{X}^{\bullet}, F\right)\right) \\
& \longrightarrow \widetilde{D R}\left(K_{Y}^{\bullet}, F\right) \quad \text { for } p \geq d_{X},
\end{aligned}
$$


where the first morphism is a filtered quasi-isomorphism as in the proof of 1.14. In fact, $\widetilde{D R}^{-1} \operatorname{Tr}_{f}$ is represented by a filtered morphism $\widetilde{D R}^{-1} f_{\bullet} \widetilde{D R}\left(K_{X}^{\bullet}, F\right) \rightarrow\left(K_{Y}^{\bullet}, F\right)$, if $K_{Y}^{\bullet}$ is chosen appropriately (cf. [S1, 2.5.1]), and we apply $\widetilde{D R}$ to it, and restrict to $F_{p}$.

3.9. Proposition. - The filtered trace morphism $\operatorname{Tr}_{f}$ exists canonically so that $\operatorname{Tr}_{g f}=\operatorname{Tr}_{g} \circ g_{\bullet} \operatorname{Tr}_{f}$ in $D F\left(\mathcal{O}_{Z}\right.$, Diff $)$ for $X \stackrel{f}{\longrightarrow} Y \stackrel{g}{\longrightarrow} Z$.

Proof. - In the analytic case, it is represented by the push-down of the distributions ( $c f .[\mathrm{S} 1,2.5 .1])$. In the algebraic case, we may assume $X, Y$ proper by Nagata-Hironaka, because the independence of the compactification follows from the functoriality for composition. If $f$ is a closed embedding of codimension $d$, we have $f_{*} \omega_{X}=\mathcal{H}_{X}^{d}\left(\omega_{Y}\right)$ and $f_{*} K_{X}^{\bullet} \stackrel{\sim}{\longrightarrow} \Gamma_{X} K_{Y}^{\bullet} \rightarrow K_{Y}^{\bullet}$. Then the filtered trace morphism is induced by the identity $f_{*}\left(\omega_{X}, F\right) \rightarrow\left(f_{*} \omega_{X}, F\right)$ with $\operatorname{Gr}_{p}^{F} f_{*} \omega_{X}=0$ for $p \neq 0$, because $f_{\bullet} \widetilde{D R}\left(\omega_{X}\left[d_{X}\right], F\right)=\widetilde{D R}\left(f_{*}\left(\omega_{X}\left[d_{X}\right], F\right)\right)(c f .[S 1,2.3])$, and $\operatorname{Gr}_{0}^{F} \operatorname{Tr}_{f}$ is the composition

$$
\omega_{X}\left[d_{X}\right]=\mathcal{E} x t_{\mathcal{O}_{Y}}^{d}\left(\mathcal{O}_{X}, \omega_{Y}\right)\left[d_{X}\right] \longrightarrow \mathcal{H}_{X}^{d}\left(\omega_{Y}\right)\left[d_{X}\right] \longrightarrow \omega_{Y}\left[d_{Y}\right] .
$$

In the case $f$ is the projection $X \times Y \rightarrow Y$, we may assume $Y=p t$, because $\omega_{X \times Y}=\omega_{X} \otimes \omega_{Y}$ and $\widetilde{D R}\left(\omega_{X \times Y}\left[d_{X}+d_{Y}\right], F\right)=\widetilde{D R}\left(\omega_{X}\left[d_{X}\right], F\right) \otimes$ $\widetilde{D R}\left(\omega_{Y}\left[d_{Y}\right], F\right)$. By Hodge theory, the filtration $F$ on $\mathbb{R} \Gamma\left(X, \widehat{D R}\left(\omega_{X}\left[d_{X}\right]\right)\right)$ is strict and $H^{0}\left(X, \widetilde{D R}\left(\omega_{X}\left[d_{X}\right]\right)\right)=\mathrm{Gr}_{0}^{F} H^{0}\left(X, \widetilde{D R}\left(\omega_{X}\left[d_{X}\right]\right)\right)=\mathbb{C}$, and we get $\operatorname{Tr}_{f}: f_{\bullet} \widetilde{D R}\left(K_{X}, F\right) \rightarrow(\mathbb{C}, F)$. In general we use the canonical factorization $X \rightarrow X \times Y \rightarrow Y$ of $f$ as in 3.1. The functoriality is clear for the composition of closed embeddings and that of projections, and it is reduced to the commutativity for

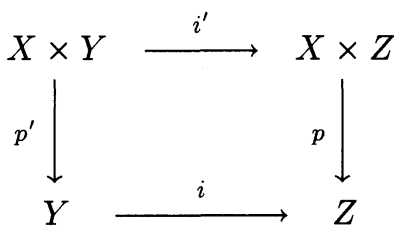

where $i$ is a closed embedding, $i^{\prime}=\mathrm{id} \times i$, and $p, p^{\prime}$ are projections. But it is also clear by definition.

3.10 Remark. - If we use the theory of mixed Hodge Modules in the algebraic case ( $c f .[\mathrm{S} 2-3])$, the filtered trace morphism is given as follows. 
For $f: X \rightarrow Y$ a proper morphism of smooth algebraic varieties, we have

$$
f_{\#}: f_{*} \mathbb{Q}_{X}^{H}\left(d_{X}\right)\left[2 d_{X}\right] \longrightarrow \mathbb{Q}_{Y}^{H}\left(d_{Y}\right)\left[2 d_{Y}\right]
$$

by the adjoint relation for $f_{!}, f^{!}$[loc. cit.]. Taking the underlying morphism of filtered $\mathcal{D}$-Modules, we get

$$
\begin{aligned}
\operatorname{Tr}_{f}: \mathbb{R} f_{*}\left(\omega_{X}\left[d_{X}\right], F\right) \longrightarrow\left(\omega_{Y}\left[d_{Y}\right], F\right) & \text { in } D^{b} F\left(\mathcal{D}_{Y}\right) \\
\operatorname{Tr}_{f}: \mathbb{R} f_{\bullet} \widetilde{D R}\left(\omega_{X}\left[d_{X}\right], F\right) \longrightarrow \widetilde{D R}\left(\omega_{Y}\left[d_{Y}\right], F\right) & \text { in } D^{b} F\left(\mathcal{O}_{Y}, \text { Diff }\right) .
\end{aligned}
$$

We can check that this trace morphism coincides with the above one in the case of closed embeddings and projections. By this definition, the check of the functoriality is easy.

3.11 TheOREM. - For $f: X \rightarrow Y$ a proper morphisms of complex manifolds, or smooth algebraic varieties, and $L^{\bullet} \in D_{g, \text { coh }}^{b}\left(\mathcal{O}_{X}, \text { Diff }\right)^{f}(c f$. 1.15), we have a canonical functor isomorphism

$$
f_{*} \mathbb{D} L^{\bullet} \stackrel{\sim}{\longrightarrow} \mathbb{D} f_{*} L^{\bullet} \quad \text { in } \quad D_{g, \text { coh }}^{b}\left(\mathcal{O}_{Y}, \text { Diff }\right)^{f} .
$$

Proof. - By 1.14, we have a $D$-quasi-isomorphism

$$
\begin{aligned}
& \mathcal{H o m}_{\mathrm{Diff}}^{f}\left(\mathbb{R} f_{\bullet} L^{\bullet}, f_{\bullet} \widetilde{D R}\left(K_{X}^{\bullet}\right)\right) \longleftarrow \\
& F_{p} \widetilde{D R}\left(\widetilde{D R}^{-1}\left(\mathcal{H o m}_{\mathrm{Diff}}^{f}\left(\mathbb{R} f_{\bullet} L^{\bullet}, f_{\bullet} \widetilde{D R}\left(K_{X}^{\bullet}\right)\right)\right)\right) \\
& \mathcal{H o m}_{\mathrm{Diff}}^{f}\left(\mathbb{R} f_{\bullet} L^{\bullet}, F_{p} \widetilde{D R}\left(\widetilde{D R}^{-1}\left(f_{\bullet} \widetilde{D R}\left(K_{X}^{\bullet}\right)\right)\right)\right)
\end{aligned}
$$

where the filtration $F$ on the differential complex

$$
\mathcal{H o m}_{\text {Diff }}^{f}\left(\mathbb{R} f_{\bullet} L^{\bullet}, f_{\bullet} \widetilde{D R}\left(K_{X}^{\bullet}\right)\right)
$$

is given by that of $f_{\bullet} \widetilde{D R}\left(K_{X}^{\bullet}\right)$, and the order of the differential morphisms is not counted here. Then the morphism (3.11.1) is given by its composition with

$$
\begin{aligned}
& f_{*} \mathrm{D} L^{\bullet}=f_{\bullet} \mathcal{H o m}_{\mathrm{Diff}}^{f}\left(L^{\bullet}, \widetilde{D R}\left(K_{X}^{\bullet}\right)\right) \longrightarrow \mathcal{H o m}_{\mathrm{Diff}}^{f}\left(\mathbb{R} f_{\bullet} L^{\bullet}, f_{\bullet} \widetilde{D R}\left(K_{X}^{\bullet}\right)\right) \\
& \mathcal{H o m}_{\mathrm{Diff}}^{f}\left(\mathbb{R} f_{\bullet} L^{\bullet}, F_{p} \widetilde{D R}\left(\widetilde{D R}{ }^{-1}\left(f_{\bullet} \widetilde{D R}\left(K_{X}^{\bullet}\right)\right)\right)\right) \\
& \stackrel{\operatorname{Tr}_{f}}{\longrightarrow} \mathcal{H o m}_{\mathrm{Diff}}^{f}\left(\mathbb{R} f_{\bullet} L^{\bullet}, \widetilde{D R}\left(K_{Y}^{\bullet}\right)\right) .
\end{aligned}
$$

томе $117-1989-\mathrm{N}^{\circ} 3$ 
To show the isomorphism of (3.11.1), we may assume $L$ is a coherent $\mathcal{O}_{X}$ Module so that $f_{*} \mathbb{D} L=f_{\bullet} \mathcal{H o m}_{\mathcal{O}_{X}}\left(L, K_{X}^{\bullet}\right), \mathbb{D} f_{*} L=\mathcal{H o m}_{\mathcal{O}_{X}}\left(\mathbb{R} f_{\bullet} L, K_{Y}^{\bullet}\right)$ by 1.17 and 1.5. Moreover they are complexes of $\mathcal{O}_{Y}$-Modules and $D R^{-1}$ is given by $\otimes_{\mathcal{O}_{Y}} \mathcal{D}_{Y}$. We define the filtration $F$ on the intermediate terms appeared in the construction of the morphism (3.11.1) as in [S1, paragraph 2], where $F$ on $L$ is trivial, i.e. $\operatorname{Gr}_{p}^{F} L=0(p \neq 0)$, and the order of the differnetial morphisms are counted so that $\mathrm{Gr}_{p}^{F}$ are defined in the category of $\mathcal{O}$-Modules. Then $\mathrm{Gr}_{p}^{F}$ of $f_{*} \mathbb{D} L, \mathbb{D} f_{*} L$ are $D$-acyclic for $p \neq 0$, and $\mathrm{Gr}_{0}^{F}$ of the morphism (3.11.1) is the composition

$$
f_{\bullet} \mathcal{H o m}_{\mathcal{O}_{Y}}\left(L, K_{X}^{\bullet}\right) \longrightarrow \mathcal{H o m}_{\mathcal{O}_{X}}\left(\mathbb{R} f_{\bullet} L, f_{\bullet} K_{X}^{\bullet}\right) \stackrel{\operatorname{Tr}_{f}}{\longrightarrow} \mathcal{H o m}_{\mathcal{O}_{Y}}\left(\mathbb{R} f_{\bullet} L, K_{Y}^{\bullet}\right)
$$

Therefore it is a quasi-isomorphism by $[\mathrm{H}],[\mathrm{RRV}]$, and a $D$-quasiisomorphism, because it is defined in $D\left(\mathcal{O}_{Y}\right)$.

3.12 Corollary. - For $M^{\bullet} \in D_{g, \text { coh }}^{b}\left(\mathcal{D}_{X}\right)$, we have a canonical functor isomorphism

$$
f_{*} \mathbb{D} M^{\bullet} \stackrel{\sim}{\longrightarrow} \mathbb{D} f_{*} M^{\bullet} \quad \text { in } D_{g, \text { coh }}^{b}\left(\mathcal{D}_{Y}\right)
$$

compatible with (3.11.1) by $\widetilde{D R}$.

(This is clear.)

3.13. Theorem. - The duality isomorphisms (3.11.1), (3.12.1) are compatible with the topological one [V2] :

$$
f_{*} \mathbb{D} K \stackrel{\sim}{\longrightarrow} \mathbb{D} f_{*} K \quad \text { in } D_{c}^{b}\left(\mathbb{C}_{Y}\right) \quad \text { or } D_{c}^{b}\left(\mathbb{C}_{Y^{\text {an }}}\right)
$$

for $K \in D_{c}^{b}\left(\mathbb{C}_{X}\right)$ or $D_{c}^{b}\left(\mathbb{C}_{X^{\text {an }}}\right)$ by For, $D R$ in the holonomic case.

Proof. - This follows from the condition for the filtered trace morphism : For $\left(\operatorname{Tr}_{f}\right)$ represents the topological trace morphism.

3.14. Remark. - If we want to define the duality isomorphism as (0.3) in the introduction, the trace morphism $\operatorname{Tr}_{f}: f_{\bullet} K_{X} \rightarrow K_{Y}$ must satisfy some condition for the compatibility with the differential of $M$. For example, if the differential $d: \mathcal{O}_{X} \rightarrow \mathcal{O}_{X}$ is given by $\xi \in f_{\bullet} \Theta_{X}$, put $\xi 1_{X \rightarrow Y}=\sum g_{i} \otimes \eta_{i} \in \mathcal{D}_{X / Y}=\mathcal{O}_{X} \otimes_{f} \bullet \mathcal{O}_{Y} f \bullet \mathcal{D}_{Y}$ locally on $Y$. Then $\operatorname{Tr}_{f}$ must satisfy

$$
\operatorname{Tr}_{f}(u \xi)=\sum \operatorname{Tr}_{f}\left(u g_{i}\right) \eta_{i} \in K_{Y}^{j} \quad \text { for } \quad u \in f_{\bullet} K_{X}^{j}
$$

In fact the general condition is

$$
\operatorname{Tr}_{f} \text { is extended to a morphism of double complex }
$$

$$
f_{\bullet} \widetilde{D R}\left(K_{X}\right) \longrightarrow \widetilde{D R}\left(K_{Y}\right)
$$


and it induces the filtered trace morphism taking the filtration associated with $\widetilde{D R}$. But $(3.14 .1-2)$ are rather difficult to realize in the level of complex. In the case $Y=$ pt, (3.14.1) implies $\operatorname{Tr}_{f}(u \xi)=0$. But $\xi: K_{X}^{i} \rightarrow$ $K_{X}^{i}$ is surjective, if $K_{X}^{i}$ is injective over $\mathcal{D}_{X}$ and $\xi \neq 0$. Therefore we can assume only the injectivity over $\mathcal{O}_{X}$.

3.15 Remark. - The duality $f_{*} \mathbb{D} M \stackrel{\sim}{\longrightarrow} \mathbb{D} f_{*} M$ induces the adjoint relation

$$
\mathcal{H o m}_{D\left(\mathcal{D}_{X}\right)}\left(M, f^{!} N\right) \stackrel{\sim}{\longrightarrow} \mathcal{H o m}_{D\left(\mathcal{D}_{Y}\right)}\left(f_{*} M, N\right)
$$

for $M \in D_{g, \text { coh }}^{b}\left(\mathcal{D}_{X}\right), N \in D^{b}\left(\mathcal{D}_{Y}\right)$ and a proper morphism $f: X \rightarrow Y$. In fact it is enough to take $H^{0} \mathbb{R} \Gamma(Y, *)$ of the tensor of $f_{*} \mathbb{D} M \stackrel{\sim}{\longrightarrow} \mathbb{D} f_{*} M$ with $\left(\omega_{Y}^{-1}\right) \otimes \mathcal{O}_{Y} N$ over $\mathcal{D}_{Y}$. This relation was used in the proof of the regularity of Hodge Modules ( $c f$. [S1]).

\section{Diagonal Pairings and Riemann-Hilbert Correspondence}

4.1.. - The Riemann-Hilbert correspondence for regular holonomic $\mathcal{D}$ Modules was proved by [K3] and [M2] (cf. [B], [Be]) in the algebraic case. In [K3] the inverse functor is constructed using the tempered distribution. In [M2], [B], [Be] the fully faithfulness of the de Rham functor (or solution) was first proved using the commutativity of $\delta^{!}$and the de Rham or solution, where $\delta: X \rightarrow X \times X$ is the diagonal embedding. But it was not so clear whether the isomorphism constructed between $\operatorname{Hom}_{D\left(\mathcal{D}_{X}\right)}\left(M^{\bullet}, N^{\bullet}\right)$ and $\operatorname{Hom}_{D\left(\mathbb{C}_{X}\right)}\left(D R\left(M^{\bullet}\right), D R\left(N^{\bullet}\right)\right)\left(\right.$ or $\left.\operatorname{Hom}_{D\left(\mathbb{C}_{X}\right)}\left(\operatorname{Sol}\left(N^{\bullet}\right), \operatorname{Sol}\left(M^{\bullet}\right)\right)\right)$ was really induced by $D R$ (or Sol), because we had to check the commutativity of some diagram which is not quite obvious. In this section we check this point using the diagonal pairings. In the algebraic case we use non quasicoherent $\mathcal{D}$-Modules.

4.2. LEMMA. - Let $X$ be a complex manifold or a smooth algebraic variety, $\delta: X \rightarrow X \times X$ the diagonal embedding, and $p r_{i}: X \times X \rightarrow X$ the projection $(i=1,2)$. Then for a right $\mathcal{D}_{X}$-Module $M$, we have a natural isomorphism

$$
M \otimes \mathcal{O}_{X} \mathcal{D}_{X}=\delta^{\bullet} \delta_{*} M
$$

as right $\mathcal{D}_{X}$-bi-Modules, where $\mathrm{pr}_{i}^{\bullet}(i=1,2)$ is used to give the first and second structure of right $\mathcal{D}_{X}$-Module on $\delta^{\bullet} \delta_{*} M$, and the first (resp. second) structure of $M \otimes_{\mathcal{O}_{X}} \mathcal{D}_{X}$ is the one associated to $\otimes_{\mathcal{O}_{X}}$ (cf. the proof of 1.7) (resp. right multiplication).

$$
\text { TOME } 117-1989-\mathrm{N}^{\circ} 3
$$


Proof. - By definition of direct image for right $\mathcal{D}$-Modules, we have a natural inclusion

$$
\phi: \delta_{\bullet} M \longrightarrow \delta_{*} M
$$

which is $\mathcal{O}_{X}$-linear for the both structure of $\delta_{*} M$, and induces a $\mathcal{D}_{X}$-linear morphism

$$
\psi: \delta . M \otimes_{\mathcal{O}_{X}} \mathcal{D}_{X} \longrightarrow \delta_{*} M
$$

using the second structure of $\delta_{*} M$. Then it is bijective by definition, and $\mathcal{D}_{X}$-linear for the first structure, because $\phi(m v)=\phi(m)\left(p r_{1}^{\bullet} v+p r_{2}^{\bullet} v\right)$ for $m \in M, v \in \Theta_{X}$ (in fact $v 1_{X \rightarrow X \times X}=1_{X \rightarrow X \times X}\left(p r_{1}^{\bullet} v+p r_{2}^{\bullet} v\right)$ where $\left.1_{X \rightarrow X \times X}=1 \otimes 1 \in \mathcal{D}_{X \rightarrow X \times X}=\mathcal{O}_{X} \otimes_{\mathcal{O}_{X \times X}} \mathcal{D}_{X \times X}\right)$.

4.3. Lemma. - For right $\mathcal{D}_{X}$-Modules $M, N$ and a right $\mathcal{D}_{X \times X}$ Module $K$ such that $\operatorname{supp} K \subset \operatorname{Im} \delta$, we have a natural isomorphism

$$
\mathcal{H o m}_{\mathcal{D}_{X}}\left(M, \mathcal{H o m}_{\mathcal{D}_{X}}\left(N, \delta^{\bullet} K\right)\right)=\mathcal{H o m}_{\mathcal{D}_{X \times X}}(M \otimes N, K)
$$

where $M \otimes N=\left(p r_{1}^{\bullet} M \otimes_{\mathbb{C}} p r_{2}^{\bullet} N\right) \otimes_{p r_{1}^{\bullet} \mathcal{D}_{X} \otimes_{\mathbb{C}} p r_{2}^{\bullet} \mathcal{D}_{X}} \mathcal{D}_{X \times X}$.

Proof. - This is clear by definition.

4.4. Corollary. - If $K$ is an injective right $\mathcal{D}_{X \times X}$-Module and $\operatorname{supp} K \subset \operatorname{Im} \delta$, then $\delta^{\bullet} K$ is injective over $\mathcal{D}_{X}$ for the both structure and $\operatorname{Hom}_{\mathcal{D}_{X}}\left(N, \delta^{\bullet} K\right)$ is an injective right $\mathcal{D}_{X}$-Module for any $N$. (Here the injectivity is considered in the not necessarily quasicoherent $\mathcal{D}$-Modules in the algebraic case).

Proof. - The assertion is clear by taking the global sections of (4.3.1), and putting $M=\mathcal{D}_{X}$ for the injectivity of $\delta^{\bullet} K$, because $\mathcal{O}_{X \times X} \otimes_{p r} \dot{\mathcal{O}}_{X} p r_{1}^{\bullet} M$ is flat over $\mathcal{O}_{X}$ by $p r_{2}^{\bullet}$ and $\boldsymbol{\nabla}$ is exact for the both factors.

Remark. - The above $K$ may not be quasicoherent if $\operatorname{dim} X \neq 0$, because the action of nonzero vector fields on $K$ is surjective by the injectivity of $K$.

4.5. Corollary. - For $M^{\bullet}, N^{\bullet} \in D^{b}\left(\mathcal{D}_{X}\right)$ and $K^{\bullet} \in D^{b}\left(\mathcal{D}_{X \times X}\right)$ such that $\operatorname{supp} \mathcal{H}^{j} K^{\bullet} \subset \operatorname{Im} \delta$, we have a natural isomorphism

$$
\begin{array}{r}
\operatorname{Hom}_{D\left(\mathcal{D}_{X}\right)}\left(M^{\bullet}, \mathbb{R} \mathcal{H o m}_{\mathcal{D}_{X}}\left(N^{\bullet}, \delta^{\bullet} K^{\bullet}\right)\right) \\
=\operatorname{Hom}_{D\left(\mathcal{D}_{X \times X}\right)}\left(M^{\bullet} \otimes N^{\bullet}, K^{\bullet}\right) .
\end{array}
$$

Proof. - Replace $K^{b}$ ullet by an injective resolution supported in $\operatorname{Im} \delta$ of $\Gamma_{\operatorname{Im} \delta}$ of its injective resolution, and apply $H^{0} \Gamma(X, *)$ to (4.3.1). 
4.6. Proposition. - For $M^{\bullet}, N^{\bullet} \in D_{\text {hol }}^{b}\left(\mathcal{D}_{X}\right)$, we have a commutative diagram

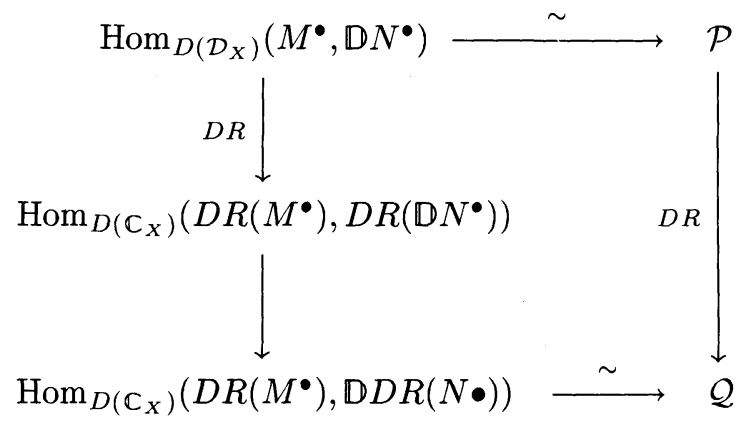

with the abreviations

$$
\begin{aligned}
& \mathcal{P}=\operatorname{Hom}_{D\left(\mathcal{D}_{X \times X}\right)}\left(M^{\bullet} \otimes N^{\bullet}, \delta_{*} \omega_{X}\left[d_{X}\right]\right), \\
& \mathcal{Q}=\operatorname{Hom}_{D\left(\mathbb{C}_{X \times X}\right)}\left(D R\left(M^{\bullet}\right) \otimes D R\left(N^{\bullet}\right), \delta_{*} \mathbb{C}_{X}\left[2 d_{X}\right]\right) .
\end{aligned}
$$

Here $\mathbb{C}_{X}, \mathbb{C}_{X \times X}$ are replaced by $\mathbb{C}_{X}$ an, $\mathbb{C}_{X^{\text {an }} \times X^{\text {an }}}$ in the algebraic case.

Proof. - The first horizontal isomorphism follows from 4.5 taking an injective resolution $K^{\bullet}$ of $\delta_{*} \omega_{X}\left[d_{X}\right]$. In the analytic case we apply $D R$ to this isomorphism, and get the second one with the commutative diagram of the assertion by the proof of 4.5 ( $c f$. the correspondence in 4.3), because $\delta^{\bullet} D R\left(K^{\bullet}\right)=D R\left(D R\left(\delta^{\bullet} K^{\bullet}\right)\right)$ is an injective resolution of $\mathbb{C}_{X}\left[2 d_{X}\right]$. Here the left vertical morphism is factorized as in the assertion by using the duality isomorphism 2.7 (cf. 2.12) :

$$
\begin{aligned}
\operatorname{DR}\left(\mathcal{H o m}_{\mathcal{D}_{X}}\left(N^{\bullet}, \delta^{\bullet} K^{\bullet}\right)\right)=\mathcal{H o m}_{\mathcal{D}_{X}}\left(N^{\bullet}, D R\left(\delta^{\bullet} K^{\bullet}\right)\right) \\
\stackrel{\sim}{\longrightarrow} \mathcal{H o m}_{\mathbb{C}_{X}}\left(D R\left(N^{\bullet}\right), D R\left(D R\left(\delta^{\bullet} K^{\bullet}\right)\right)\right) .
\end{aligned}
$$

In the algebraic case we apply the above argument to $\mathrm{An}(M)$, etc. (cf. 2.9), and use 2.9 with the compatibility of 4.5 with An by taking an injective resolution $K_{\text {an }}^{\bullet}$ of $\operatorname{An}\left(K^{\bullet}\right)$.

4.7. Remark. - In the case $M^{\bullet}, N^{\bullet}$ have regular holonomic cohomologies, the right vertical morphism $D R$ is an isomorphism by the commutativity of $\delta^{*}, \delta_{*}$ with $D R$, and the duality. In fact, we can replace $M^{\bullet} \otimes N^{\bullet}$ in $\mathcal{P}$ and $D R\left(M^{\bullet}\right) \otimes D R\left(N^{\bullet}\right)$ in $\mathcal{Q}$ by $\delta_{*} \delta^{*}\left(M^{\bullet} \otimes N^{\bullet}\right)$ and $\delta_{*} D R\left(\delta^{*}\left(M^{\bullet} \otimes N^{\bullet}\right)\right)$. Then the assertion is reduced to

$$
\begin{aligned}
D R: \operatorname{Hom}_{D\left(\mathcal{D}_{X}\right)} & \left(\delta^{*}\left(M^{\bullet} \otimes N^{\bullet}\right), \omega_{X}\left[d_{X}\right]\right) \\
& \stackrel{\sim}{\longrightarrow} \operatorname{Hom}_{D\left(\mathbb{C}_{X}\right)}\left(D R\left(\delta^{*}\left(M^{\bullet} \otimes N^{\bullet}\right)\right), \mathbb{C}_{X}\left[2 d_{X}\right]\right)
\end{aligned}
$$

TOME $117-1989-\mathrm{N}^{\circ} 3$ 
and follows from $D R \mathbb{D} \stackrel{\sim}{\longrightarrow} \mathbb{D} D R(c f .2 .7)$ in the analytic case. In the algebraic case we have to use also the commutativity of $D R$ (or An) with the direct image onto a point, where the regularity at infinity is essentially used. Here we need also the fully faithfulness of $D^{b}\left(\mathcal{D}_{X}\right)_{q \text { coh }} \longrightarrow D^{b}\left(\mathcal{D}_{X}\right)$, where the first (resp. second) is the bounded derived category of quasicoherent (resp. not necessarily quasicoherent) $\mathcal{D}_{X}$-Modules, $c f$. [B]. This property is equivalent to the following (equivalent) left (resp. right) effaceability : for any quasicoherent $\mathcal{D}_{X}$-Modules $M, N$ and $e \in \mathcal{E} x t^{k}(M, N)$, there exists a surjection $u: M^{\prime} \rightarrow M$ (resp. an injection $v: N \rightarrow N^{\prime}$ ) such that $u^{*} e=0$ in $\mathcal{E} x t^{k}\left(M^{\prime}, N\right)$ (resp. $v_{*} e=0$ in $\mathcal{E} x t^{k}\left(M, N^{\prime}\right)$ ) for $k>0$. In the affine case the left effaceability is easily checked using a surjection from a free Module, and the general case is reduced to this case using the right effaceability and an affine covering with the adjunction for $j^{*}, j_{*}$, $c f$. [loc. cit.]. Then by the commutativity of the diagram we get the isomorphism of the left vertical morphism $D R$ and the fully faithfulness of the de Rham functor. It seems that the check of the commutativity of the above diagram is not so easy if $D R$ is replaced by Sol ( $c f .2 .8$ ), because Sol is contravariant and the diagonal pairing is not preserved by Sol as in the proof of 4.6 .

4.8. Remark. - We can also define the duality isomorphism using the diagonal pairings. For $M=\mathbb{D} N, N \in D_{g, \text { coh }}^{b}\left(\mathcal{D}_{X}\right)$, we have a canonical element

$$
c \in \operatorname{Hom}_{D\left(\mathcal{D}_{X \times X}\right)}\left(M \otimes N, \delta_{*} \omega_{X}\left[d_{X}\right]\right)
$$

corresonding to id $\in \operatorname{Hom}_{D\left(\mathcal{D}_{X}\right)}(M, \mathbb{D} N)$. By the direct image we get

$$
\begin{gathered}
f_{*} c \in \operatorname{Hom}_{D\left(\mathcal{D}_{Y \times Y}\right)}\left(f_{*} M \otimes f_{*} N, \delta_{*} f_{*} \omega_{X}\left[d_{X}\right]\right) \\
\operatorname{Tr}_{f} \circ f_{*} c \in \operatorname{Hom}_{D\left(\mathcal{D}_{Y \times Y}\right)}\left(f_{*} M \otimes f_{*} N, \delta_{*} \omega_{Y}\left[d_{Y}\right]\right) .
\end{gathered}
$$

For the perfectness of $\operatorname{Tr}_{f} \circ f_{*} c$, i.e. the isomorphism of the corresponding morphism $f_{*} \mathbb{D} N \rightarrow \mathbb{D} f_{*} N$, we use the filtered trace morphism $\operatorname{Tr}_{f}$ : $f_{*}\left(\omega_{X}\left[d_{X}\right], F\right) \rightarrow\left(\omega_{Y}\left[d_{Y}\right], F\right)$ such that $\operatorname{Gr}_{0}^{F} \operatorname{Tr}_{f}$ is the analytic, or algebraic, trace morphism $\operatorname{Tr}_{f}: \mathbb{R} f_{\bullet} \omega_{X}\left[d_{X}\right] \rightarrow \omega_{Y}\left[d_{Y}\right]$ as in 3.11. Here note that $\operatorname{Gr}_{0}^{F}\left(f_{*} \omega_{X}\left[d_{X}\right]\right)=\mathbb{R} f_{\bullet} \omega_{X}\left[d_{X}\right]$ and $\operatorname{Gr}_{0}^{F} \omega_{Y}\left[d_{Y}\right]=\omega_{Y}\left[d_{Y}\right]$ are invariant by the closed embeddings (in particular by $\delta_{*}$ ), because $0=\min \left\{p: \operatorname{Gr}_{p}^{F} \omega_{X} \neq 0\right\}$ (same for $Y$ ).

4.9. Remark. - It would be possible to define the duality isomorphism (0.1) using the trace morphism of bimodules :

$$
\begin{aligned}
\operatorname{Tr}_{f}: \mathbb{R} f_{\bullet}\left(\left(\left(\omega_{X}\left[d_{X}\right] \otimes \mathcal{D}_{X}\right) \otimes_{\mathcal{D}_{X}}^{\mathrm{L}} \mathcal{D}_{X \rightarrow Y}\right)\right. & \left.\otimes_{\mathcal{D}_{X}}^{\mathrm{L}} \mathcal{D}_{X \rightarrow Y}\right) \\
& \longrightarrow \omega_{Y}\left[d_{Y}\right] \otimes \mathcal{D}_{Y},
\end{aligned}
$$


(cf. [Sc1-2]). But to do so, we have to show a nontrivial isomorphism

$$
\begin{aligned}
\left(\omega_{X} \otimes_{f} \bullet \mathcal{O}_{Y}\right. & \left.f^{\bullet} \mathcal{D}_{Y}\right) \otimes_{\mathcal{D}_{X}}^{\mathrm{L}}\left(\mathcal{O}_{X} \otimes_{f} \bullet \mathcal{O}_{Y} f^{\bullet} \mathcal{D}_{Y}\right) \\
& \simeq\left(\omega_{X} \otimes_{\mathcal{D}_{X}}^{\mathrm{L}}\left(\mathcal{O}_{X} \otimes_{f} \bullet \mathcal{O}_{Y} f^{\bullet} \mathcal{D}_{Y}\right)\right) \otimes_{f} \bullet \mathcal{O}_{Y} f^{\bullet} \mathcal{D}_{Y}
\end{aligned}
$$

as right $f^{\bullet} \mathcal{D}_{Y}$-bi-Modules, and the relation with the trace morphism for $\mathcal{O}$-Modules and relative $\mathcal{D}$-Modules becomes nontrivial. As a consequence, it is not so clear that the duality isomorphism proved in [loc. cit.] is really induced by (4.9.1). Here we have to take the representative of $\mathbb{R} f_{\bullet}\left(M \otimes_{\mathcal{D}_{X}} \mathcal{D}_{X \rightarrow Y}\right)$, etc. to check the assertion. In fact, $\mathbb{R} \mathcal{H o m}_{\mathcal{D}_{X}}\left(M, \omega_{X}\left[d_{X}\right] \otimes \mathcal{D}_{X}\right)$ cannot be defined without taking an injective resolution of $\omega_{X}\left[d_{X}\right] \otimes \mathcal{D}_{X}$, and $M \otimes_{\mathcal{D}_{X}} \mathcal{D}_{X \rightarrow Y}$ without a (flat) resolution of $M$ or $\mathcal{D}_{X \rightarrow Y}$, etc. and we have to check some commutativity of diagram in the level of complexes (compare to paragraph 3). In [Sc2], the isomorphism (4.9.2) seems to be reduced to the isomorphism

$$
\begin{aligned}
\left(\mathcal{O}_{X} \otimes_{f} \bullet \mathcal{O}_{Y} f^{\bullet} \mathcal{D}_{Y}\right) \otimes_{\mathcal{O}_{X}} & \left(\mathcal{O}_{X} \otimes_{f} \bullet \mathcal{O}_{Y} f^{\bullet} \mathcal{D}_{Y}\right) \\
& \simeq\left(\mathcal{O}_{X} \otimes_{f} \bullet \mathcal{O}_{Y} f^{\bullet} \mathcal{D}_{Y}\right) \otimes_{f} \bullet \mathcal{O}_{Y} f^{\bullet} \mathcal{D}_{Y}
\end{aligned}
$$

as right $f^{\bullet} \mathcal{D}_{Y}$-bi- and left $\mathcal{D}_{X}$-Modules, where the left $\mathcal{D}_{X}$-Module structure of $\mathcal{D}_{X \rightarrow Y}=\mathcal{O}_{X} \otimes_{f} \bullet \mathcal{O}_{Y} f^{\bullet} \mathcal{D}_{Y}$ is used for $\otimes_{\mathcal{O}_{X}}$ in the left hand side, and its right $f^{\bullet} \mathcal{D}_{Y}$-Module structure for $\otimes_{f} \bullet \mathcal{O}_{Y}$ in the right. Then we can prove it directly by $f \otimes P \otimes g \otimes Q \mapsto(f g \otimes 1 \otimes Q) t(P)$, where $t$ means the right $f^{\bullet} \mathcal{D}_{Y}$-Module structure associated with the tensor of right and left $f^{\bullet} \mathcal{D}_{Y}$-Modules over $f^{\bullet} \mathcal{O}_{Y}$ ( $c f$. the proof of 1.7). Note that we can also use 4.8 to define (4.9.1), because (4.9.1) should be the direct image by $\delta$ of the trace morphism.

Here it should be also noted that the involution 1.7 must be used in the proof of id $\stackrel{\sim}{\longrightarrow} \mathbb{D}^{2}$ in [loc. cit.].

\section{BIBLIOGRAPHY}

[B] Borel (A.). - Algebraic D-Modules. - Academic Press, 1987.

[Be] Bernstein (J.). - Algebraic theory of D-Modules. - Preprint, 1983 .

TOME $117-1989-\mathrm{N}^{\circ} 3$ 
[BO] Berthelot (P.) and Ogus (A.). - Notes on Crystalline cohomology. Princeton University Press, 1978.

[G] Grothendieck (A.). - Éléments de géométrie algébrique III. Publ. Math. IHES, t. 11, 1961.

[H] Hartshorne (R.). - Residues and duality. - Lecture Notes in Math., t. 20, 1966.

[K1] Kashiwara (M.). - On the maximally overdetermined system of linear differential equations I, Publ. RIMS, Kyoto Univ., t. 10, 1975, p. $563-579$.

[K2] Kashiwara (M.). $-B$-functions and holonomic systems, Invent. Math., t. 38, 1976, p. 33-53.

[K3] Kashiwara (M.). - The Riemann-Hilbert Problem for Holonomic Systems, Publ. RIMS, Kyoto Univ., t. 20, 1984, p. 319-365.

[KK] Kashiwara (M.) and Kawai (T.). - On holonomic system of microdifferential equations III, Publ. RIMS, Kyoto Univ., t. 17, 1981, p. 813-979.

[M1] Meвkhout (Z.). - Théorème de bidualité pour les $\mathcal{D}_{X}$-Modules holonomes, Ark. Mat., t. 20, 1982, p. 111-124.

[M2] Meвкhout (Z.). - Une autre équivalence de catégorie, Compositio Math., t. 51, 1984, p. 63-88.

[RRV] Ramis (J.-P.), Ruget (G.) et Verdier (J.-L.). - Dualité relative en géométrie analytique complexe, Invent. Math., t. 13, 1971, p. 261283.

[S1] Saito (M.). - Modules de Hodge polarisables, Publ. RIMS, Kyoto Univ., t. 24, 1988, p. 849-995.

[S2] Saito (M.). - Mixed Hodge Modules, Preprint RIMS-585.

[S3] Saito (M.). - Introduction to mixed Hodge Modules, Preprint RIMS-605 (to appear in Astérisque).

[Sc1] Schneiders (J.-P.). - Un théorème de dualité relative pour les modules différentiels, C.R. Acad. Sc. Paris, t. 303, 1986, p. 235-238.

[Sc2] Schneiders (J.-P.). - Dualité pour les modules différentiels, dissertation. - Univ. Liège, $1986-1987$.

[V1] Verdier (J.L.). - Catégories dérivées, SGA 4 1/2, Lecture Notes in Math., t. 569, 1977, p. 262-311.

[V2] Verdier (J.L.). - Dualité dans la cohomologie des espaces localement compacts, Séminaire Bourbaki, n⿳0300, 1966, Benjamin. 